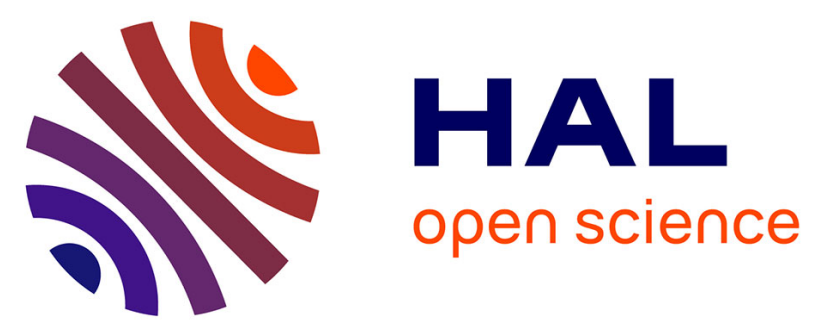

\title{
Seasonal variations of the phenolic constituents in bilberry (Vaccinium myrtillus L.) leaves, stems and fruits, and their antioxidant activity
}

Oana-Crina Bujor, Carine Le Bourvellec, Irina Volf, Valentin I. Popa, Claire Dufour

\section{To cite this version:}

Oana-Crina Bujor, Carine Le Bourvellec, Irina Volf, Valentin I. Popa, Claire Dufour. Seasonal variations of the phenolic constituents in bilberry (Vaccinium myrtillus L.) leaves, stems and fruits, and their antioxidant activity. Food Chemistry, 2016, 213, pp.58-68. 10.1016/j.foodchem.2016.06.042 . hal-02640817

\section{HAL Id: hal-02640817 https://hal.inrae.fr/hal-02640817}

Submitted on 28 May 2020

HAL is a multi-disciplinary open access archive for the deposit and dissemination of scientific research documents, whether they are published or not. The documents may come from teaching and research institutions in France or abroad, or from public or private research centers.
L'archive ouverte pluridisciplinaire HAL, est destinée au dépôt et à la diffusion de documents scientifiques de niveau recherche, publiés ou non, émanant des établissements d'enseignement et de recherche français ou étrangers, des laboratoires publics ou privés. 


\title{
Seasonal variations of the phenolic constituents in bilberry (Vaccinium myrtillus L.) leaves, stems and fruits, and their antioxidant activity
}

\author{
Oana-Crina Bujor ${ }^{\mathrm{a}, \mathrm{b}}$, Carine Le Bourvellec ${ }^{\mathrm{b}}$, Irina Volf ${ }^{\mathrm{a}}$, Valentin I. Popa ${ }^{\mathrm{a}}$, Claire Dufour ${ }^{\mathrm{b}, *}$ \\ a “Gheorghe Asachi” Technical University of Iasi, Faculty of Chemical Engineering and Environmental Protection, 700050 Iasi, Romania \\ ${ }^{\mathrm{b}}$ UMR408 Safety and Quality of Plant Products, INRA, University of Avignon, F-84000 Avignon, France
}

Keywords:

Polyphenols

Antiradical capacity

Vegetation periods

UPLC/MS

Folin-Ciocalteu

DPPH, Thioacidolysis

\begin{abstract}
A B S T R A C T
The seasonal variations of the content and diversity of phenolic compounds, as well as the antioxidant activity of leaves, stems and fruits of bilberry collected in May, July and September, were evaluated for two consecutive years. UPLC/MS ${ }^{\mathrm{n}}$ analyses showed the predominance of anthocyanins in fruits, caffeic acid derivatives in leaves whereas flavanol oligomers represented more than half of the phenolic compounds in stems. Thioacidolysis revealed degrees of polymerization between 2 and 4 and (-)epicatechin as the main flavanol unit. The sum of the phenolic compounds by UPLC was highly correlated with the total polyphenol content and the antioxidant activity in the DPPH test for all the extracts except for May leaves. The latter were relatively rich in $p$-coumaric acid derivatives. Seasonal effects were more marked for leaves, which exhibited higher antioxidant activities and phenolic contents in July and September when these parameters were at their highest in July for stems.
\end{abstract}

\section{Introduction}

Bilberry (Vaccinium myrtillus L.), also known as European blueberry, whortleberry, and huckleberry, is a wild shrub, which can be found in the mountains and forests of Europe, and the north of America. Fruits and aerial parts of bilberry are known as a natural source of food and beverage, due to its richness in nutritional and bioactive compounds and are also consumed as dietary supplements and pharmaceutical products for health benefits. Bilberry fruit extracts have been studied for the prevention and treatment of chronic pathologies, such as diabetes, cardiovascular disease and obesity (Erlund et al. 2008; Mauray et al., 2010; Mykkänen et al., 2014; Rouanet et al., 2010). Anti-inflammatory properties of bilberry fruits are central to this health protection.

Leaves and stems of bilberry are used as herbal tea, the most consumed form, or hydro-alcoholic extract in traditional herbal medicine and have also been shown to exhibit antibacterial and antioxidant activities (Vučić et al., 2013). These benefits are attributed to the high content in phenolic compounds (flavonoids, phenolic acids and proanthocyanidins) in bilberry leaves (Martz, Jaakola, Julkunen-Tiitto, \& Stark, 2010). The in vitro and in vivo biological activities of phenolic compounds from natural sources

* Corresponding author at: INRA, UMR SQPOV, 228 rte de l'Aérodrome, 84914 Avignon Cedex 9, France.

E-mail address: claire.dufour@avignon.inra.fr (C. Dufour). involve application as antioxidants, antibacterial and anticarcinogenic agents, allelochemicals, and plant growth regulators (Bujor, Talmaciu, Volf, \& Popa, 2015).

The quality and quantity of phenolic compounds in $V$. myrtillus L. are generally influenced by the parts of the plant to be used, the stage of growth, the environmental conditions and genetic factors (Akerström, Jaakola, Bång, \& Jäderlund, 2010; Jovančević et al., 2011; Martz et al., 2010; Mikulic-Petkovsek, Schmitzer, Slatnar, Stampar, \& Veberic, 2015; Uleberg et al., 2012). It may appear that higher phenolic contents are favoured by northern latitudes, altitude and a sunny environment. In bilberry fruits, high amounts of anthocyanins and hydroxycinnamic acid derivatives as well as low amounts of flavonols, proanthocyanidins and coumaroyl iridoids were identified (Mikulic-Petkovsek et al., 2015). In contrast, leaves are known to contain, in decreasing levels, hydroxycinnamic acids, flavonol glycosides and proanthocyanidins but also cinchonains and iridoids in unknown amounts (Liu et al., 2014; Martz et al., 2010). A comparative study conducted by Teleszko and Wojdyło (2015) showed that phenolic compounds were found in a markedly higher content in leaves than in fruits, in agreement with the strongest antioxidant capacity displayed by leaves compared to fruits.

To date, most works have focused on the study of a single morphological part of the bilberry plant, fruits most commonly, leaves and stems sometimes. In this context, the primary aim of this study is to simultaneously assess the seasonal variations of phenolic 
compounds and antioxidant activity in leaves, stems, and fruits of bilberry, with the purpose of determining which harvest period or part of the Vaccinium plant can be selected as the best source of phenolic antioxidants. Indeed, a good knowledge of the phenolic compound distribution in the various plant tissues of bilberry can play a key role in guiding their fields of use, either as nutraceuticals or food additives. Moreover, this knowledge is of the upmost importance to investigate the mechanisms involved in the health effect of these different polyphenol extracts. Thus, contents in total polyphenols were assessed globally by the Folin-Ciocalteu method or specifically by UPLC/MS and were tentatively correlated with the antioxidant capacity in the DPPH test. Finally, an original analysis of the oligomeric proanthocyanidins is proposed, addressing both the degree of polymerisation and flavanol unit constitution.

\section{Methodology}

\subsection{Materials}

\subsubsection{Bilberry samples}

Leaves and stems of wild bilberry (V. myrtillus L.) were collected in May, July and September, while the fruits were collected in July at the ripening stage during the years 2013 and 2014, from mountains near Borca (Neamt, Romania, coordinates: $47^{\circ} 11^{\prime} 34^{\prime \prime} \mathrm{N}$ and $25^{\circ} 47^{\prime} 8^{\prime \prime} \mathrm{E}$ ).

Fresh bilberry fruits were frozen at $-24^{\circ} \mathrm{C}$, then lyophilised in a Christ Alpha 1-4 LSC (Osterode, Germany) freeze dryer for 3 days and finally ground for $25 \mathrm{~s}$ at $2000 \mathrm{rpm}$ in a knife mill (Retsch Grindomix GM 200) to a fine powder. Leaves and stems of bilberry were dried at room temperature, in the shade, for 7 days. After drying, leaves were manually separated from stems, ground as above and sieved to a final particle size $<0.315 \mathrm{~mm}$. Before extraction, the residual moisture of grinded samples was determined using a RADWAG MAX 50/1 moisture analyzer (RADWAG Balances \& Scales, Radom, Poland). Residual moistures between 7\% and 9.5\% were found for all plant materials.

\subsubsection{Chemicals and solvents}

Chemicals: Anhydrous sodium carbonate, 37\% hydrochloric acid, Folin and Ciocalteu's phenol reagent, DPPH (1,1-diphenyl-2picrylhydrazyl), Trolox (6-hydroxy-2,5,7,8-tetramethylchroman-2 -carboxylic acid) and toluene- $\alpha$-thiol were purchased from Sigma-Aldrich (Saint-Quentin Fallavier, France). Polyphenol standards, gallic acid, chlorogenic acid, $p$-coumaric acid, (+)-catechin, (-)-epicatechin were from Sigma-Aldrich; quercetin-3-Oglucoside, quercetin-3-O-rhamnoside, quercetin-3-O-galactoside, procyanidin B2, procyanidin A2, (-)-epigallocatechin and cyanidin-3-O-galactoside were purchased from Extrasynthese (Genay, France); procyanidin C1 was extracted from apple fruits (Malus domestica Borkh.) of the Kermerrien variety as described previously (Watrelot, Renard, \& Le Bourvellec, 2015).

Solvents: $96 \%$ ethanol was purchased from S.C. Chemical Company (Iași, Romania), HPLC-MS grade methanol and acetonitrile from Fisher Scientific (Illkirch, France), formic acid from Merck (Darmstadt, Germany), and glacial acetic acid from Merck (Fontenay Sous Bois, France). Ultrapure water (resistivity $18.2 \mathrm{M} \Omega . \mathrm{cm}^{-1}$ at $25^{\circ} \mathrm{C}$ ) was obtained with a Milli-Q water purification system (Millipore, Bedford, MA).

\subsection{Extraction of phenolic compounds}

To $1 \mathrm{~g}$ of ground bilberry leaf, stem and fruit samples placed in an extraction vial fitted with a condenser was added $30 \mathrm{~mL}$ of $1 \%$ aqueous citric acid. Next, the mixture was extracted in a Milestone START S microwave oven for synthesis, at a microwave power of
$300 \mathrm{~W}$, for $7 \mathrm{~min}$ and a temperature of $40^{\circ} \mathrm{C}$ (Zheng et al., 2013). Additionally, the fruit samples were extracted with $55 \%$ aqueous ethanol $(v / v)$ under the same extraction conditions. The extracts were filtered and then the volume of each sample was adjusted to $30 \mathrm{~mL}$ prior to the determination of the total phenolic content (TPC). The extract solutions were freeze-dried and the dry extracts (DE) stored at $4{ }^{\circ} \mathrm{C}$ before use. Triplicate extractions were made for each sample. Dry matter (DM) refers to the initially ground dry sample after correction from the residual water.

\subsection{Qualitative and quantitative analyses of phenolic compounds}

For UPLC/MS analyses, freshly prepared solutions of leaf and stem dry extracts $(10 \mathrm{mg} / \mathrm{mL})$ in water were directly used. For the fruit dry extract, purification was first conducted to eliminate sugars and organic acids that could interfere in the analysis of phenolic compounds. Solutions of fruit extracts at $20 \mathrm{mg} / \mathrm{mL}$ prepared in $1 \%$ aqueous $\mathrm{HCl}(v / v)$ were purified by elution on C18 Sep-Pak Plus mini-columns (360 mg; Waters, Milford, MA). The C18 cartridge was first conditioned with two column volumes of $0.01 \%$ $\mathrm{HCl}$ in methanol followed by three volumes of $0.01 \%$ aqueous $\mathrm{HCl}$ $(v / v)$ to remove remaining methanol. Secondly, the extracts were injected onto the mini-column and then the cartridge was washed with two volumes of $0.01 \%$ aqueous $\mathrm{HCl}$. Finally, the phenolic compounds were eluted with $0.01 \% \mathrm{HCl}$ in methanol. The phenolic fractions were immediately subjected to UPLC/MS analyses.

Separation and identification of phenolic compounds were performed using a Waters ACQUITY UPLC chromatograph (Waters, Milford, MA) coupled to a UV-Vis diode-array detector and an HCT ultra ion trap mass spectrometer equipped with electrospray ionisation (Mane, Loonis, Juhel, Dufour, \& Malien-Aubert, 2011).

Polyphenol standards in $\mathrm{MeOH}$, except for cyanidin-3galactoside, which was further acidified with $1 \% \mathrm{HCl}(\mathrm{v} / \mathrm{v})$, were used for 6-point calibrations. Other phenolic compounds were quantified as follows: caffeic acid derivatives and sinapic acid hexoside as chlorogenic acid ( $325 \mathrm{~nm}$ ), coumaric acid derivatives as $p$ coumaric acid (330 nm), quercetin glycosides as quercetin-3-Ogalactoside $(350 \mathrm{~nm})$, A-type dimers as procyanidin A2 $(280 \mathrm{~nm})$, B-type dimers and cinchonains II as procyanidin B2 $(280 \mathrm{~nm})$, Atype and B-type trimers as procyanidin C1 $(280 \mathrm{~nm})$, cinchonains I as (-)-epicatechin, 3,4-dihydroxyphenylpropionic acid hexoside as $(+)$-catechin and anthocyanins as cyanidin-3-O-galactoside $(520 \mathrm{~nm})$. The flavanol monomers were calculated as the sum of (-)-epicatechin and (-)-epigallocatechin or cinchonain I, while the flavanol oligomers were reported as the sum of all the dimers, trimers, and cinchonains II. Injected volumes were $3 \mu \mathrm{L}$ for phenolic compounds and $1 \mu \mathrm{L}$ for anthocyanins. All samples were injected in triplicate from independently prepared solutions of dry extracts.

\subsection{Analysis of procyanidins using thioacidolysis}

Procyanidin analysis was performed after thioacidolysis in the presence of toluene- $\alpha$-thiol using a method adapted from Le Bourvellec et al. (2011). Procyanidins were characterised by their subunit composition and their average degree of polymerisation (mDP). The HPLC apparatus was a Shimadzu LC-20AD equipped with a SPD-M20A DAD detector and an RF-10AXL fluorescence detector (Shimadzu, Kyoto, Japan). Separations were achieved using a Lichrocart column $(250 \mathrm{~mm} \times 4 \mathrm{~mm}$ i.d, Lichrospher PR$185 \mathrm{~mm}$ ) with a guard column (Lichrospher PR-18 $5 \mathrm{~mm}$ column; Merck, Darmstadt, Germany) operated at $30^{\circ} \mathrm{C}(20 \mu \mathrm{L}$ injected). (+)-Catechin benzyl thioether was quantified at $280 \mathrm{~nm}$ as (+)catechin whereas (-)-epicatechin benzyl thioether was quantified as (-)-epicatechin. In samples containing anthocyanins, (+)catechin and (-)-epicatechin were specifically identified and 
quantified by their excitation-emission energy (278 $\mathrm{nm}$ and $360 \mathrm{~nm}$ ), in order to avoid overlapping peaks due to anthocyanin absorbance at $280 \mathrm{~nm}$.

\subsection{Antioxidant activity by applying spectrophotometric methods}

\subsubsection{Total phenolic contents by the Folin Ciocalteu method}

The total phenolic content (TPC) of the extract solutions was determined by the Folin-Ciocalteu spectrophotometric method described by Hainal, Ignat, Volf, and Popa (2011). An aliquot of $1 \mathrm{~mL}$ of the diluted extract (1:50 for leaves and stems, and 1:25 for fruits) was mixed with $0.5 \mathrm{~mL}$ of the Folin-Ciocalteu reagent ( $2 \mathrm{M}), 2 \mathrm{~mL}$ of $10 \%$ aq. $\mathrm{Na}_{2} \mathrm{CO}_{3}$ and $5 \mathrm{~mL} \mathrm{H}_{2} \mathrm{O}$. Then, the mixture was left for $90 \mathrm{~min}$ in the dark at room temperature. Absorbance was measured at $765 \mathrm{~nm}$ (CINTRA 101 UV-VIS spectrometer; GBC Scientific Equipment Ltd., Dandenong, Australia) using a mixture of water and reagents as a blank. The results were expressed as mg of gallic acid equivalents per gram of dry matter (mg GAE/ $\mathrm{g}$ DM) after correction from residual moisture. Triplicates from independent extract solutions were analysed.

\subsubsection{DPPH (2,2-diphenyl-1-picrylhydrazyl) radical scavenging test}

The DPPH test was adapted from a method developed by Goupy, Dufour, Loonis, and Dangles (2003). Small volumes (25 $\mu \mathrm{L})$ from dry extracts freshly prepared in water $(10 \mathrm{mg} / \mathrm{mL})$ were added to $2 \mathrm{~mL}$ of a $0.2 \mathrm{mM}$ solution of DPPH in methanol. The decay of the absorbance at $515 \mathrm{~nm}$ (HP 8453 diode-array spectrometer, optical path length $=1 \mathrm{~cm}$ ) was recorded for $30 \mathrm{~min}$ at $25^{\circ} \mathrm{C}$ under constant magnetic stirring $(1250 \mathrm{rpm})$. The results were expressed as micromoles of Trolox equivalents (TE) per gram of dry extract using Trolox calibration curves. All determinations were carried out three to four times and independent extract solutions were used each time.

\subsection{Statistical analyses}

Results are expressed as the mean \pm standard deviation (SD). Significant differences at a $95 \%$ confidence interval were assessed through the analysis of ANOVA with Tukey-Kramer post hoc test using XLStat software (version 2008.3.02; Addinsoft SARL, Paris, France).

\section{Results and discussion}

\subsection{Phenolic profile and content of bilberry extracts from UPLC/MS} analyses

In all the morphological parts of bilberry 106 phenolic compounds were tentatively identified, with 62 in leaf, 73 in stem and 40 in fruit extracts (Table 1). Additionally, 17 phenolic compounds were found only in leaves, 32 only in stems, and 9 only in fruits. Maximum absorption wavelength, molecular ion and fragmentation pattern in MS were used for structure assessment in the absence of standards.

\subsubsection{Caffeic acid derivatives}

In leaves, caffeic acid derivatives are present in 3- to 10-fold higher levels compared to coumaric acid derivatives, whereas there is no content difference in stems in these two hydroxycinnamic acid derivatives (Table 2). Caffeic acid derivatives were principally found in leaves as caffeic acid esterified with quinic acid, shikimic acid and monotropein or esterified/etherified with a hexose moiety.
Caffeoylquinic acids grafted with a hexosyl group were represented by two diversely polar molecules (9 and 51). Both of them were newly identified in bilberry. Compound $\mathbf{9}$ with fragment ions at $m / z 353$ and 191 was assigned as 5-O-caffeoylquinic acid-4'-Ohexoside, based on the fragmentation pattern similar to that of 5-O-caffeoylquinic acid and the hypsochromic shift in the spectrum. Compound 51 displayed a major fragment ion at $\mathrm{m} / \mathrm{z} 341$ (caffeic acid hexoside) and fragments at $\mathrm{m} / \mathrm{z} 191$ and 173 (typical of 4-O-caffeoylquinic acid). The four compounds displaying a parent ion at $m / z 707$ (MS) and major fragments at $m / z 353\left(\mathrm{MS}^{2}\right)$ and at $\mathrm{m} / \mathrm{z} 191\left(\mathrm{MS}^{3}\right)$ are presumably caffeoylquinic acid derivatives $(\mathbf{2 0}, \mathbf{2 2}, \mathbf{2 4}$ and 29). The presence of two further hydrogens when comparing with the structure of a caffeoylquinic acid covalent dimer as well as a $\lambda_{\max }$ at $282 \mathrm{~nm}$ suggest that the $\alpha, \beta-$ unsaturated double bond in caffeic acid is no longer present. A fragment at $m / z 515$ could be interpreted as an additional caffeoyl or hexosyl unit on caffeoylquinic acid. Related caffeoylquinic acid derivatives $(\mathbf{5 0}, \mathbf{5 6})$, sharing a parent ion at $m / z 705$ and a sole fragment ion at $m / z 513$, display a maximal absorption wavelength at $320 \mathrm{~nm}$. They could be caffeoylquinic acid covalent dimers or result from the oxidation of the previous caffeoylquinic acid derivatives with $m / z$ 707. Another caffeoylquinic acid derivative (74), with a parent ion at $\mathrm{m} / \mathrm{z} 381$ and fragmentation ions at $\mathrm{m} / \mathrm{z}$ 191, 179, 161 and 135 typical for caffeoylquinic acid, was identified in leaves (Ieri, Martini, Innocenti, \& Mulinacci, 2013) and newly described in fruit.

Caffeic acid can be covalently bound to glycosyl residues in two different manners through esterification or etherification. Etherification of the 4-hydroxyl group of caffeic acid led to two isomers of caffeic acid-4- $O-\beta$-D-hexoside (9 and 17) whose structures are supported by the lack of clear absorption at $\lambda_{\max } 320 \mathrm{~nm}$ (Mane et al., 2011). The first isomer was present in leaves and fruits while the second was in fruits only. By analogy with the fragmentations of p-coumaroyl malonylhexosides (83 and 94 on one side, and 96 and $\mathbf{9 7}$ on the other side), compounds $\mathbf{6 8}$ and $\mathbf{8 7}$ were assessed as caffeoyl malonylhexosides. Caffeoyl malonylhexosides, which have been identified in all the morphological parts of bilberry, are newly named in this study (Ieri et al., 2013).

\subsubsection{Coumaric acid derivatives}

In leaves and stems, two p-coumaroylquinic acids (30 and 44) were assigned respectively to the cis and trans forms of 5-pcoumaroylquinic acid, based on their major fragment ion at $\mathrm{m} / \mathrm{z}$ 191 (quinic acid) resulting from the loss of $p$-coumaric acid and the hypsochromic shift in the UV-Vis spectrum of $\mathbf{4 4}$ (Clifford, Knight, Johnston \& Kuhnert, 2003). Additionally, four hexosides of $p$-coumaric acid $(\mathbf{7}, \mathbf{8}, 14$ and 21$)$ were detected in the various morphological parts. Compound 8 displays a $\lambda_{\max }$ at $295 \mathrm{~nm}$, which is characteristic of the electronic density modification induced by the glycosylation at the $\mathrm{O}-4$ position (Chanforan, Loonis, Mora, Caris-Veyrat, \& Dufour, 2012). Derivatives with a $\lambda_{\max }$ at $310 \mathrm{~nm}$ are esters of $p$-coumaric acid. $p$-Coumaric acid hexosides can be further acylated by acetic acid and malonic acid. Indeed, two $p$ coumaroyl diacetylhexosides with $m / z 409$ ( 78 and 92) were tentatively identified in all the morphological parts based on fragment ions at $m / z 325$ (loss of 2 acetyl groups) and $m / z 163$ typical of $p$ coumaric acid. Also, three $p$-coumaroyl triacetylhexosides with $m / z 451(\mathbf{9 8}, 102$ and 105) were only observed in leaves. Two of them were characterised by fragment ions at $m / z 367$ (loss of 2 acetyl groups) and $m / z 245$ (loss of both acetyl and $p$-coumaroyl groups), while the other isomer displayed a first fragment at $\mathrm{m} / \mathrm{z}$ 341.

Putative malonylated derivatives comprise four $p$-coumaroyl malonylhexosides, which are present in leaves, stems, and fruits, 
Version définitive du manuscrit publiée dans / Final version of the manuscript published in :

Food Chemistry (2016), Vol. 213, p. 58-68, DOI: 10.1016/j.foodchem.2016.06.042

Journal homepage : www.elsevier.com/locate/foodchem

Table 1

Phenolic compounds identified by UPLC/ESI-MS ${ }^{\mathrm{n}}$ in leaf, stem and fruit extract of bilberry.

\begin{tabular}{|c|c|c|c|c|c|c|c|c|c|c|c|}
\hline \multirow[t]{2}{*}{ No. } & \multirow{2}{*}{$\begin{array}{l}\mathrm{t}_{\mathrm{R}} \\
(\min )\end{array}$} & \multirow{2}{*}{$\begin{array}{l}\lambda_{\max } \\
(\mathrm{nm})\end{array}$} & \multirow{2}{*}{$\begin{array}{l}{[\mathrm{M}-\mathrm{H}]^{-}} \\
(m / z)\end{array}$} & \multirow[t]{2}{*}{$\mathrm{MS}^{2}$ fragments $(m / z)$} & \multirow[t]{2}{*}{ Proposed structure } & \multicolumn{3}{|l|}{2013} & \multicolumn{3}{|l|}{2014} \\
\hline & & & & & & May & July & Sept. & May & July & Sept. \\
\hline 1 & 1.65 & 270 & 305 & $287,261,219, \underline{179}, 165,125$ & (+)-Gallocatechin ${ }^{c}$ & $\mathrm{~S}$ & $\mathrm{~S}$ & $\mathrm{~S}$ & $\mathrm{~S}$ & $\mathrm{~S}$ & $\mathrm{~S}$ \\
\hline 2 & 1.7 & 240 & 389 & $227,209,183,165,139$ & Monotropein ${ }^{c}$ & $\mathrm{~S}$ & $\mathrm{~S}$ & $\mathrm{~S}$ & $\mathrm{~S}$ & $\mathrm{~S}$ & $\mathrm{~S}$ \\
\hline 3 & 1.9 & 288 & 329 & $\overline{167}, \underline{152 ; \mathrm{MS}^{3}}[167]: 152,123,108$ & $\begin{array}{l}\text { Hydroxymethoxybenzoic acid - } \\
\text { hexose }\end{array}$ & $S^{a}$ & $\mathrm{~S}^{\mathrm{a}}$ & $S^{a}$ & $S^{a}$ & $\mathrm{~S}$ & $\mathrm{~S}$ \\
\hline 4 & 2.1 & 278 & 315 & $\underline{153}, 123$ & Dihydroxybenzoic acid - hexose & $S^{a}$ & $\mathrm{~S}$ & $\mathrm{~S}$ & $\mathrm{~S}$ & $\mathrm{~S}$ & $\mathrm{~S}$ \\
\hline 5 & 2.45 & 278 & 451 & $405, \underline{289}, 245,161$ & (epi)Catechin derivative (1) & $S$ & $S$ & $S$ & $S$ & $\mathrm{~S}$ & $\mathrm{~S}$ \\
\hline 6 & 2.55 & $\begin{array}{l}290 \text { sh, } \\
310\end{array}$ & 447 & $315,271,207, \underline{152}$ & $\begin{array}{l}\text { Dihydroxybenzoic acid - hexose- } \\
\text { pentose }\end{array}$ & $S^{a}$ & $\mathrm{~S}$ & $\mathrm{~S}$ & $\mathrm{~S}$ & $\mathrm{~S}$ & $\mathrm{~S}$ \\
\hline 7 & 2.6 & $\begin{array}{l}290 \text { sh, } \\
310\end{array}$ & 325 & $307,187,163,119$ & p-Coumaroylhexoside (1) & $\mathrm{L}, \mathrm{S}^{\mathrm{a}}$ & $\begin{array}{l}\mathrm{L}^{\mathrm{a}}, \mathrm{S}, \mathrm{F}_{\mathrm{H}_{2} \mathrm{O}} \\
\mathrm{F}_{\mathrm{EtOH}}\end{array}$ & $\mathrm{L}^{\mathrm{a}}, \mathrm{S}$ & $\mathrm{L}, \mathrm{S}$ & $\begin{array}{l}\mathrm{L}^{\mathrm{a}}, \mathrm{S}, \mathrm{F}_{\mathrm{H}_{2} \mathrm{O}} \\
\mathrm{F}_{\mathrm{EtOH}}\end{array}$ & $\mathrm{L}, \mathrm{S}$ \\
\hline 8 & 2.7 & $\begin{array}{l}295 \\
306 \mathrm{sh}\end{array}$ & 325 & 163, 119 & $p$-Coumaric acid-4-O-hexoside $(1)^{\mathrm{h}}$ & $\mathrm{L}$ & $\mathrm{L}$ & $\mathrm{L}$ & $\mathrm{L}$ & $\mathrm{L}^{\mathrm{a}}$ & $\mathrm{L}$ \\
\hline 9 & 2.8 & $\begin{array}{l}290, \\
320 \mathrm{sh}\end{array}$ & 515 & $\underline{353}, 191$ & $\begin{array}{l}\text { 5-O-Caffeoylquinic acid-4'-O- } \\
\text { hexoside }^{\mathrm{i}}\end{array}$ & $\mathrm{L}$ & $\mathrm{L}$ & $\mathrm{L}$ & $\mathrm{L}^{\mathrm{a}}$ & $\mathrm{L}$ & $\mathrm{L}$ \\
\hline 10 & & & 341 & $\underline{179}, 135$ & Caffeic acid-4-O- $\beta$-D-hexoside $(1)^{\mathrm{h}}$ & $\mathrm{L}$ & $\begin{array}{l}\mathrm{L}, \mathrm{F}_{\mathrm{H}_{2} \mathrm{O}} \\
\mathrm{F}_{\mathrm{EtOH}} \mathrm{a}\end{array}$ & $\mathrm{L}$ & $\mathrm{L}$ & $\begin{array}{l}\mathrm{L}, \mathrm{F}_{\mathrm{H}_{2} \mathrm{O}} \\
\mathrm{F}_{\mathrm{EtOH}}\end{array}$ & $\mathrm{L}$ \\
\hline 11 & 2.9 & & 593 & $\underline{575}, 467,441, \underline{423}, 305,287,273$ & $\begin{array}{l}\text { (epi)Gallocatechin-(epi)catechin } \\
\text { dimer (1) }\end{array}$ & $\mathrm{S}^{\mathrm{a}}$ & $\mathrm{S}$ & $\mathrm{S}$ & $\mathrm{S}^{\mathrm{a}}$ & $\mathrm{S}$ & $\mathrm{S}$ \\
\hline 12 & 3.05 & 278 & 863 & $\begin{array}{l}711,575 \mathrm{MS}^{3}[575]: 499, \underline{489}, 451, \\
289,287,245\end{array}$ & A-type trimer (1) & $\mathrm{S}$ & $\mathrm{S}$ & $\mathrm{S}$ & $\mathrm{S}$ & $\mathrm{S}$ & $\mathrm{S}$ \\
\hline 13 & 3.1 & 279 & 405 & $\underline{289}, 179$ & (epi)Catechin derivative (2) & - & $\mathrm{L}^{\mathrm{a}}$ & $\mathrm{L}$ & $\mathrm{L}^{\mathrm{a}}$ & - & $\mathrm{L}$ \\
\hline 14 & 3.3 & $\begin{array}{l}290 \text { sh, } \\
312\end{array}$ & 325 & $307, \underline{187,} 163$ & p-Coumaroylhexoside (2) & $\mathrm{L}, \mathrm{S}^{\mathrm{a}}$ & $\mathrm{L}, \mathrm{S}$ & $\mathrm{L}, \mathrm{S}$ & $\mathrm{L}, \mathrm{S}$ & $\mathrm{L}, \mathrm{S}$ & $\mathrm{L}, \mathrm{S}$ \\
\hline 15 & 3.6 & 270 & 305 & $287,261, \underline{221}, 219, \underline{179}, 165,125$ & $(-)$-Epigallocatechin $(\mathrm{std})^{\mathrm{c}}$ & $\mathrm{S}$ & $\begin{array}{l}\mathrm{S}, \mathrm{F}_{\mathrm{H}_{2} \mathrm{O}}{ }^{\mathrm{a}} \\
\mathrm{F}_{\mathrm{EtOH}}{ }^{\mathrm{a}}\end{array}$ & $\mathrm{S}$ & $\begin{array}{l}\mathrm{S}, \mathrm{F}_{\mathrm{H}_{2} \mathrm{O}}{ }^{\mathrm{a}} \\
\mathrm{F}_{\mathrm{EtOH}}{ }^{\mathrm{a}}\end{array}$ & $\mathrm{S}$ & $\mathrm{S}$ \\
\hline 16 & 3.7 & 278 & 577 & $559,451,425, \underline{407}, 289,245$ & Procyanidin B1 (std) & $\mathrm{S}$ & $\mathrm{S}$ & $\mathrm{S}$ & $\mathrm{S}^{\mathrm{a}}$ & $\mathrm{S}^{\mathrm{a}}$ & $\mathrm{S}^{\mathrm{a}}$ \\
\hline 17 & & & 341 & 179,135 & Caffeic acid-4-O- $\beta$-D-hexoside $(2)^{i}$ & & $\mathrm{~F}_{\mathrm{H}_{2} \mathrm{O}}, \mathrm{F}_{\mathrm{EtOH}}$ & & & $\mathrm{F}_{\mathrm{H}_{2} \mathrm{O}}, \mathrm{F}_{\mathrm{EtOH}}$ & \\
\hline 18 & 3.8 & $\begin{array}{l}295 \mathrm{sh}, \\
324\end{array}$ & 353 & 191 & 5-O-Caffeoylquinic-acid (std) & $\mathrm{L}, \mathrm{S}$ & $\begin{array}{l}\mathrm{L}, \mathrm{S}, \mathrm{F}_{\mathrm{H}_{2} \mathrm{O}} \\
\mathrm{F}_{\mathrm{EtOH}}\end{array}$ & $\mathrm{L}, \mathrm{S}$ & $\mathrm{L}, \mathrm{S}$ & $\begin{array}{l}\mathrm{L}, \mathrm{S}, \mathrm{F}_{\mathrm{H}_{2} \mathrm{O}} \\
\mathrm{F}_{\mathrm{EtOH}}\end{array}$ & $\mathrm{L}, \mathrm{S}$ \\
\hline 19 & 3.9 & & 593 & $575,467,441, \underline{423}, 305,287,245$ & $\begin{array}{l}\text { (epi)Gallocatechin-(epi)catechin } \\
\text { dimer (2) }\end{array}$ & $\mathrm{S}$ & $\mathrm{S}$ & $\mathrm{S}$ & $\mathrm{S}$ & $\mathrm{S}$ & $\mathrm{S}$ \\
\hline \multirow[t]{2}{*}{20} & 4.2 & 282 & 707 & $533,515,463,393,341, \underline{323}, 297$ & Caffeoylquinic acid derivative (1) & $\mathrm{L}$ & $\mathrm{L}$ & $\mathrm{L}$ & $\mathrm{L}$ & $\mathrm{L}$ & $\mathrm{L}^{\mathrm{a}}$ \\
\hline & & & & $\mathrm{MS}^{3}[353]: 191$ & & $\mathrm{~L}$ & $\mathrm{~L}$ & $\mathrm{~L}^{\mathrm{a}}$ & $\mathrm{L}$ & $\mathrm{L}$ & $\mathrm{L}$ \\
\hline 21 & 4.3 & & 325 & $\underline{163}, 119$ & p-Coumaric acid hexoside (2) & $S^{a}$ & $S^{a}$ & $\mathrm{~S}$ & $\mathrm{~S}$ & $\mathrm{~S}$ & $\mathrm{~S}$ \\
\hline \multirow[t]{2}{*}{22} & 4.55 & 282 & 707 & $\begin{array}{l}533,515,463,359, \underline{353}, 323,321, \\
295\end{array}$ & Caffeoylquinic acid derivative (2) & $\mathrm{L}$ & $\mathrm{L}$ & $\mathrm{L}$ & $\mathrm{L}$ & $\mathrm{L}$ & $\mathrm{L}$ \\
\hline & & & & $\mathrm{MS}^{3}[353]: 191$ & & $\mathrm{~L}^{\mathrm{a}}$ & $\mathrm{L}$ & $\mathrm{L}$ & $\mathrm{L}$ & $\mathrm{L}$ & $\mathrm{L}$ \\
\hline 23 & & & 343 & $298,221,181,161,137$ & $\begin{array}{l}\text { 3,4-Dihydroxyphenylpropionic acid } \\
\text { hexoside }^{\mathrm{h}}\end{array}$ & $\mathrm{S}$ & $\mathrm{S}$ & $\mathrm{S}$ & $\mathrm{S}$ & $\mathrm{S}$ & $\mathrm{S}$ \\
\hline \multirow[t]{2}{*}{24} & 4.65 & 282 & 707 & $\begin{array}{l}\underline{533}, 515, \underline{463}, 393,359, \underline{323}, 297, \\
271,219\end{array}$ & Caffeoylquinic acid derivative (3) & $\mathrm{L}$ & $\mathrm{L}$ & $\mathrm{L}$ & $\mathrm{L}^{\mathrm{a}}$ & $\mathrm{L}$ & $\mathrm{L}$ \\
\hline & & & & $\begin{array}{l}\text { MS }^{3}[353]: ~ 323,297,289,231, \underline{191} \\
179,173\end{array}$ & & $\mathrm{~L}^{\mathrm{a}}$ & $\mathrm{L}$ & $\mathrm{L}$ & $\mathrm{L}^{\mathrm{a}}$ & $\mathrm{L}$ & $\mathrm{L}$ \\
\hline 25 & 5.15 & 278 & 577 & $559,531,451, \underline{425}, 407,289$ & B-type dimer (2) & $L^{a}, S$ & $\mathrm{~L}, \mathrm{~S}$ & $\mathrm{~L}^{\mathrm{a}}, \mathrm{S}$ & $\mathrm{L}^{\mathrm{a}}, \mathrm{S}$ & $\mathrm{L}^{\mathrm{a}}, \mathrm{S}$ & $\mathrm{L}^{\mathrm{a}}, \mathrm{S}$ \\
\hline 26 & & & 451 & $\underline{289}, 245,161$ & Cinchonain I $(1)^{\mathrm{c}}$ & $\mathrm{S}^{\mathrm{a}}$ & $\mathrm{S}$ & S & $\mathrm{S}^{\mathrm{a}}$ & $\mathrm{S}$ & $\mathrm{S}$ \\
\hline 27 & 5.25 & & 385 & $\overline{223}$ & Sinapic acid hexoside & $\mathrm{S}$ & $\mathrm{S}^{\mathrm{a}}$ & $\mathrm{S}$ & S & $\mathrm{S}$ & $\mathrm{S}$ \\
\hline 28 & 5.7 & 278 & 865 & & B-type trimer (1) & $S^{a}$ & $\mathrm{~S}^{\mathrm{a}}$ & $S^{a}$ & $\mathrm{~S}^{\mathrm{a}}$ & $\mathrm{S}^{\mathrm{a}}$ & $\mathrm{S}^{\mathrm{a}}$ \\
\hline \multirow[t]{2}{*}{29} & & 282 & 707 & $533,513,489,353,323$ & Caffeoylquinic acid derivative (4) & $\mathrm{L}^{\mathrm{a}}$ & $\mathrm{L}$ & $\mathrm{L}$ & $\mathrm{L}^{\mathrm{a}}$ & $\mathrm{L}^{\mathrm{a}}$ & $\mathrm{L}$ \\
\hline & & & & $\operatorname{MS}^{3}$ [353]: 191 & & $\mathrm{~L}$ & $\mathrm{~L}$ & $\mathrm{~L}^{\mathrm{a}}$ & $\mathrm{L}^{\mathrm{a}}$ & $\mathrm{L}$ & $\mathrm{L}$ \\
\hline 30 & 5.75 & $\begin{array}{l}\text { 290sh, } \\
310\end{array}$ & 337 & $\underline{191}, 163$ & 5-p-Coumaroylquinic acide,g & $\mathrm{L}, \mathrm{S}^{\mathrm{a}}$ & $\mathrm{L}, \mathrm{S}$ & $\mathrm{L}, \mathrm{S}^{\mathrm{a}}$ & $\mathrm{L}, \mathrm{S}$ & $\mathrm{L}, \mathrm{S}$ & $\mathrm{L}, \mathrm{S}$ \\
\hline 31 & 6.2 & $\begin{array}{l}\text { 290sh, } \\
312\end{array}$ & 353 & 191 & 5-Caffeoylquinic acid (cis) ${ }^{\mathrm{h}}$ & $\mathrm{L}$ & $\mathrm{L}$ & $\mathrm{L}$ & $\mathrm{L}$ & $\mathrm{L}$ & $\mathrm{L}$ \\
\hline 32 & 6.3 & 278 & 577 & $559,451, \underline{425}, 407,289,245$ & Procyanidin B2 (std) (3) & $\mathrm{L}^{\mathrm{a}}, \mathrm{S}$ & $\mathrm{L}, \mathrm{S}$ & $\mathrm{L}, \mathrm{S}$ & $\mathrm{S}$ & $\mathrm{L}, \mathrm{S}$ & $\mathrm{L}^{\mathrm{a}}, \mathrm{S}$ \\
\hline 33 & 6.4 & 278 & 865 & $\begin{array}{l}847,739,713,695,587,577,575 \\
451,425,407,289,287,\end{array}$ & B-type trimer (2) & $L^{a}, S$ & $\mathrm{~L}, \mathrm{~S}$ & L, S & $S^{\mathrm{a}}$ & $\mathrm{L}^{\mathrm{a}}, \mathrm{S}$ & $\mathrm{S}$ \\
\hline 34 & 6.85 & 278 & 289 & $\underline{245}, 205,125$ & $(-)$-Epicatechin $(\mathrm{std})^{\mathrm{c}, \mathrm{e}, \mathrm{f}, \mathrm{g}}$ & $L^{a}, S$ & $\begin{array}{l}\mathrm{L}, \mathrm{S}, \mathrm{F}_{\mathrm{H}_{2} \mathrm{O}} \mathrm{a} \\
\mathrm{F}_{\mathrm{EtOH}}{ }^{\mathrm{a}}\end{array}$ & $\mathrm{L}, \mathrm{S}$ & $\begin{array}{l}\mathrm{L}, \mathrm{S}, \mathrm{F}_{\mathrm{H}_{2} \mathrm{O}}{ }^{\mathrm{a}} \\
\mathrm{F}_{\mathrm{EtOH}}{ }^{\mathrm{a}}\end{array}$ & L, S & $\mathrm{L}, \mathrm{S}$ \\
\hline 35 & 7.0 & & 335 & $\underline{179}, 135$ & Caffeoylshikimic acid ${ }^{\mathrm{c}, \mathrm{e}}$ & $\mathrm{L}$ & $\mathrm{L}^{\mathrm{a}}$ & $\mathrm{L}$ & $\mathrm{L}$ & $\mathrm{L}^{\mathrm{a}}$ & $\mathrm{L}$ \\
\hline 36 & 7.3 & 278 & 881 & $\begin{array}{l}863,755,711,593,575,467,423 \\
305,287\end{array}$ & $\begin{array}{l}\text { (epi)Gallocatechin-(epi)catechin- } \\
\text { (epi)catechin trimer }\end{array}$ & $\mathrm{S}$ & $\mathrm{S}$ & $\mathrm{S}$ & $\mathrm{S}$ & $\mathrm{S}$ & $\mathrm{S}$ \\
\hline 37 & & & 451 & $\underline{289}, 245$ & Cinchonain I (2) & - & $\mathrm{S}^{\mathrm{a}}$ & $S^{a}$ & $S^{a}$ & $S^{a}$ & $\mathrm{~S}$ \\
\hline 38 & 7.35 & & 739 & $\begin{array}{l}721 \\
329, \\
287\end{array}$ & Cinchonain II $(1)^{\mathrm{c}}$ & $S^{a}$ & S & $S^{a}$ & $S^{a}$ & $S^{a}$ & $S^{a}$ \\
\hline 39 & 7.55 & 278 & 879 & $\frac{727}{285}, 709,559,467,411,305,287$ & $\begin{array}{l}\text { A-type trimer of (epi)gallocatechin- } \\
\text { (epi)catechin-(epi)catechin }\end{array}$ & $S$ & $S$ & $S$ & $S^{a}$ & $S^{a}$ & $\mathrm{~S}$ \\
\hline 40 & 7.60 & 278 & $576^{\mathrm{b}}$ & $\begin{array}{l}567, \underline{500}, 491,451,407,289,287 \\
245\end{array}$ & B-type tetramer (1) & $\mathrm{S}$ & $\mathrm{L}^{\mathrm{a}}, \mathrm{S}$ & $\mathrm{L}^{\mathrm{a}}, \mathrm{S}^{\mathrm{a}}$ & $\mathrm{S}^{\mathrm{a}}$ & $\mathrm{L}^{\mathrm{a}}, \mathrm{S}$ & $\mathrm{L}^{\mathrm{a}}, \mathrm{S}^{\mathrm{a}}$ \\
\hline
\end{tabular}


Version définitive du manuscrit publiée dans / Final version of the manuscript published in :

Food Chemistry (2016), Vol. 213, p. 58-68, DOI: 10.1016/j.foodchem.2016.06.042

Journal homepage : www.elsevier.com/locate/foodchem

Table 1 (continued)

\begin{tabular}{|c|c|c|c|c|c|c|c|c|c|c|c|}
\hline \multirow[t]{2}{*}{ No. } & \multirow{2}{*}{$\begin{array}{l}\mathrm{t}_{\mathrm{R}} \\
(\mathrm{min})\end{array}$} & \multirow{2}{*}{$\begin{array}{l}\lambda_{\max } \\
(\mathrm{nm})\end{array}$} & \multirow{2}{*}{$\begin{array}{l}{[\mathrm{M}-\mathrm{H}]^{-}} \\
(m / z)\end{array}$} & \multirow[t]{2}{*}{ MS $^{2}$ fragments $(m / z)$} & \multirow[t]{2}{*}{ Proposed structure } & \multicolumn{3}{|l|}{2013} & \multicolumn{3}{|l|}{2014} \\
\hline & & & & & & May & July & Sept. & May & July & Sept. \\
\hline 41 & 7.80 & 278 & 863 & $\frac{711}{287}, 693,573,531,451,411,289$ & A-type trimer (2) & $\mathrm{S}$ & $\mathrm{S}$ & $\mathrm{S}^{\mathrm{a}}$ & $\mathrm{S}^{\mathrm{a}}$ & $\mathrm{S}$ & $\mathrm{S}$ \\
\hline 42 & 7.90 & 278 & 577 & $\begin{array}{l}559,541,533,451, \underline{439}, 425,393 \\
329,289,245\end{array}$ & B-type dimer (4) & $\mathrm{S}$ & $\mathrm{S}$ & $\mathrm{S}^{\mathrm{a}}$ & - & $\mathrm{S}$ & $\mathrm{S}$ \\
\hline 43 & 8.10 & & $576^{\mathrm{b}}$ & $\begin{array}{l}567,500, \underline{491}, 451 ; 407,289,287, \\
245\end{array}$ & B-type tetramer (2) & $\mathrm{S}$ & $\mathrm{L}, \mathrm{S}$ & $\mathrm{L}, \mathrm{S}$ & $\mathrm{L}^{\mathrm{a}}, \mathrm{S}$ & $\mathrm{L}^{\mathrm{a}}, \mathrm{S}$ & $\mathrm{L}^{\mathrm{a}}, \mathrm{S}$ \\
\hline 44 & 8.25 & $\begin{array}{l}285 \\
305 \mathrm{sh}\end{array}$ & 337 & $\underline{191}, 163$ & 5-p-Coumaroylquinic acid (cis) & $\mathrm{L}$ & $\mathrm{L}$ & $\mathrm{L}^{\mathrm{a}}$ & $\mathrm{L}$ & $\mathrm{L}$ & $\mathrm{L}$ \\
\hline 45 & 9.05 & 278 & 863 & $\begin{array}{l}\frac{711}{289}, 693,573,559,531,451,411 \\
\text {, }\end{array}$ & A-type trimer (3) & $\mathrm{S}$ & $\mathrm{S}$ & $\mathrm{S}$ & $\mathrm{S}$ & $\mathrm{S}$ & $\mathrm{S}$ \\
\hline 46 & 9.50 & 278 & 865 & $\begin{array}{l}847,739,713, \underline{695}, 577,543 \\
451,449,425,407,287\end{array}$ & B-type trimer (3) & $\mathrm{L}^{\mathrm{a}}, \mathrm{S}$ & $\mathrm{L}, \mathrm{S}$ & $\mathrm{L}, \mathrm{S}$ & $\mathrm{L}^{\mathrm{a}}, \mathrm{S}$ & $\mathrm{L}, \mathrm{S}$ & $\mathrm{L}^{\mathrm{a}}, \mathrm{S}$ \\
\hline 47 & 9.60 & & 575 & $\begin{array}{l}413, \underline{395}, 377,351,287,266,204 \\
165\end{array}$ & A-type dimer (1) & $\mathrm{L}, \mathrm{S}^{\mathrm{a}}$ & $\mathrm{L}^{\mathrm{a}}, \mathrm{S}^{\mathrm{a}}$ & $\mathrm{L}^{\mathrm{a}}, \mathrm{S}^{\mathrm{a}}$ & $\mathrm{L}, \mathrm{S}^{\mathrm{a}}$ & $\mathrm{L}^{\mathrm{a}}, \mathrm{S}^{\mathrm{a}}$ & $\mathrm{L}^{\mathrm{a}}, \mathrm{S}^{\mathrm{a}}$ \\
\hline 48 & 9.70 & 278 & 593 & $575,467,441, \underline{423}, 305,287$ & $\begin{array}{l}\text { (epi)Gallocatechin-(epi)catechin } \\
\text { dimer (3) }\end{array}$ & $\mathrm{S}$ & $\mathrm{S}$ & $\mathrm{S}$ & $\mathrm{S}$ & $\mathrm{S}$ & $\mathrm{S}$ \\
\hline 49 & 9.80 & & 577 & 559, 451, 425, 407, 289, 287 & B-type dimer (5) & $S$ & $\mathrm{~S}$ & $S^{a}$ & $S^{a}$ & $\mathrm{~S}$ & $S$ \\
\hline 50 & 10.00 & 320 & 705 & 513 & Caffeoylquinic acid derivative (5) & $\mathrm{L}$ & $\mathrm{L}$ & $\mathrm{L}^{\mathrm{a}}$ & $\mathrm{L}$ & $\mathrm{L}^{\mathrm{a}}$ & $\mathrm{L}^{\mathrm{a}}$ \\
\hline 51 & 10.35 & 278 & 515 & $\underline{341}, 323,297,281,255,191,173$ & Caffeoylquinic acid hexoside (2) & $\mathrm{L}^{\mathrm{a}}$ & $\mathrm{L}$ & $\mathrm{L}$ & $\mathrm{L}^{\mathrm{a}}$ & $\mathrm{L}^{\mathrm{a}}$ & $\mathrm{L}^{\mathrm{a}}$ \\
\hline 52 & 10.45 & 278 & 575 & $520, \underline{499}, 490,452,423,289,245$ & A-type dimer (2) & $\mathrm{S}$ & $\mathrm{S}$ & $S^{a}$ & S & S & $\mathrm{S}$ \\
\hline $\begin{array}{l}53 \\
54\end{array}$ & 10.60 & $\begin{array}{l}320 \\
278\end{array}$ & $\begin{array}{l}705 \\
359\end{array}$ & $\begin{array}{l}513 \\
197,153\end{array}$ & $\begin{array}{l}\text { Caffeoylquinic acid derivative (6) } \\
\text { Syringic acid hexoside }\end{array}$ & $\mathrm{L}$ & $\begin{array}{l}\mathrm{L}^{\mathrm{a}} \\
\mathrm{F}_{\mathrm{H}_{2} \mathrm{O}}, \mathrm{F}_{\mathrm{EtOH}}\end{array}$ & $\mathrm{L}^{\mathrm{a}}$ & $\mathrm{L}$ & $\begin{array}{l}\mathrm{L}^{\mathrm{a}} \\
\mathrm{F}_{\mathrm{H}_{2} \mathrm{O}}, \mathrm{F}_{\mathrm{EtOH}}\end{array}$ & $\mathrm{L}^{\mathrm{a}}$ \\
\hline 55 & 10.65 & 278 & $576^{\mathrm{b}}$ & $\begin{array}{l}559,521,500,491,451,413,289, \\
287,245\end{array}$ & B-type tetramer (3) & $\mathrm{S}$ & $\mathrm{L}, \mathrm{S}$ & $\mathrm{L}, \mathrm{S}$ & $\mathrm{L}^{\mathrm{a}}, \mathrm{S}$ & $\mathrm{L}^{\mathrm{a}}, \mathrm{S}$ & $\mathrm{L}^{\mathrm{a}}, \mathrm{S}$ \\
\hline 56 & 10.95 & & 595 & $475,463,445,343, \underline{300}, 271,255$ & Quercetin pentosyl hexoside & $\mathrm{S}$ & $\mathrm{S}$ & $\mathrm{S}$ & $\mathrm{S}$ & $\mathrm{S}$ & S \\
\hline 57 & 11.00 & $\begin{array}{l}295 \mathrm{sh}, \\
324\end{array}$ & 551 & $507, \underline{389}, 371, \underline{345}, 327, \underline{179}$ & Caffeoyl monotropein ${ }^{\mathrm{e}}$ & $\mathrm{L}$ & $\mathrm{L}^{\mathrm{a}}, \mathrm{F}_{\mathrm{EtOH}}$ & $\mathrm{L}^{\mathrm{a}}$ & $\mathrm{L}$ & $\mathrm{F}_{\mathrm{H}_{2} \mathrm{O}}, \mathrm{F}_{\mathrm{EtOH}}$ & - \\
\hline 58 & 11.05 & 278 & 865 & $\begin{array}{l}847,739, \frac{713}{245}, 695,577,575,451 \\
407,287,245\end{array}$ & B-type trimer (4) & $\mathrm{S}^{\mathrm{a}}$ & $\mathrm{S}$ & $\mathrm{S}^{\mathrm{a}}$ & $\mathrm{S}^{\mathrm{a}}$ & $\mathrm{S}^{\mathrm{a}}$ & $\mathrm{S}^{\mathrm{a}}$ \\
\hline 59 & 11.3 & 278 & 739 & $\begin{array}{l}721,629, \underline{587}, 569,435,417,339 \\
289\end{array}$ & Cinchonain II (2) & $\mathrm{L}^{\mathrm{a}}, \mathrm{S}$ & $\mathrm{L}, \mathrm{S}$ & $\mathrm{L}, \mathrm{S}$ & $\mathrm{L}^{\mathrm{a}}, \mathrm{S}$ & $\mathrm{L}, \mathrm{S}$ & L, S \\
\hline 60 & 11.7 & 278 & 451 & $\underline{341}, 217$ & Cinchonain I (3) & $\mathrm{L}^{\mathrm{a}}, \mathrm{S}$ & $\mathrm{L}, \mathrm{S}$ & $\mathrm{L}, \mathrm{S}$ & $\mathrm{L}^{\mathrm{a}}, \mathrm{S}$ & $\mathrm{L}, \mathrm{S}$ & $\mathrm{L}, \mathrm{S}$ \\
\hline 61 & 11.95 & $\begin{array}{l}254 \\
350\end{array}$ & 477 & 301 & Quercetin hexuronide (1) & $\mathrm{L}, \mathrm{S}$ & $\mathrm{L}, \mathrm{S}$ & $\mathrm{L}, \mathrm{S}$ & $\mathrm{L}, \mathrm{S}$ & $\mathrm{L}, \mathrm{S}$ & $\mathrm{L}, \mathrm{S}$ \\
\hline 62 & & & 463 & 301 & Quercetin-3-O-galactoside (std) ${ }^{g}$ & $\mathrm{~L}, \mathrm{~S}$ & $\begin{array}{l}\mathrm{L}, \mathrm{S}, \mathrm{F}_{\mathrm{H}_{2} \mathrm{O}} \\
\mathrm{F}_{\mathrm{EtOH}}\end{array}$ & $\mathrm{L}, \mathrm{S}$ & $\mathrm{L}, \mathrm{S}$ & $\begin{array}{l}\mathrm{L}, \mathrm{S}, \mathrm{F}_{\mathrm{H}_{2} \mathrm{O}} \\
\mathrm{F}_{\mathrm{EtOH}}\end{array}$ & $\mathrm{L}, \mathrm{S}$ \\
\hline 63 & & & 535 & $491,371,329,311,267,191,163$ & p-Coumaroyl monotropein $(1)^{c, e, g}$ & $\mathrm{~L}, \mathrm{~S}$ & $\begin{array}{l}\mathrm{L}^{\mathrm{a}}, \mathrm{S}, \mathrm{F}_{\mathrm{H}_{2} \mathrm{O}} \\
\mathrm{F}_{\mathrm{EtOH}}\end{array}$ & $\mathrm{L}^{\mathrm{a}}, \mathrm{S}^{\mathrm{a}}$ & $L^{a}, S^{a}$ & $\begin{array}{l}\mathrm{L}^{\mathrm{a}}, \mathrm{S}, \mathrm{F}_{\mathrm{H}_{2} \mathrm{O}} \\
\mathrm{F}_{\mathrm{EtOH}}\end{array}$ & $\mathrm{L}^{\mathrm{a}}, \mathrm{S}$ \\
\hline 64 & 12.25 & $\begin{array}{l}255 \\
352\end{array}$ & 477 & 301 & Quercetin hexuronide $(2)^{\mathrm{g}}$ & $\mathrm{L}, \mathrm{S}$ & $\begin{array}{l}\mathrm{L}, \mathrm{S}, \mathrm{F}_{\mathrm{H}_{2} \mathrm{O}} \\
\mathrm{F}_{\mathrm{EtOH}}\end{array}$ & $\mathrm{L}, \mathrm{S}$ & $\mathrm{L}, \mathrm{S}$ & $\begin{array}{l}\mathrm{L}, \mathrm{S}, \mathrm{F}_{\mathrm{H}_{2} \mathrm{O}} \\
\mathrm{F}_{\mathrm{EtOH}}\end{array}$ & $\mathrm{L}, \mathrm{S}$ \\
\hline 65 & 12.50 & $\begin{array}{l}279 \\
307\end{array}$ & 455 & $\underline{309}, 291,163,145$ & $p$-Coumaric acid derivative $\mathrm{e}^{\mathrm{e}}$ & $\mathrm{S}$ & $\mathrm{S}$ & $\mathrm{S}$ & $\mathrm{S}$ & $\mathrm{S}$ & $\mathrm{S}$ \\
\hline 66 & 12.70 & $\begin{array}{l}254 \\
354\end{array}$ & 477 & 301 & Quercetin hexuronide (3) & $\mathrm{L}$ & $\mathrm{L}^{\mathrm{a}}$ & $\mathrm{L}$ & $\mathrm{L}$ & $\mathrm{L}$ & $\mathrm{L}$ \\
\hline 67 & & & 463 & 301 & Quercetin-3-O-glucoside (std) & $\mathrm{L}$ & $\begin{array}{l}\mathrm{L}, \mathrm{F}_{\mathrm{H}_{2} \mathrm{O}} \\
\mathrm{F}_{\mathrm{EtOH}}\end{array}$ & $\mathrm{L}$ & $\mathrm{L}$ & $\begin{array}{l}\mathrm{L}, \mathrm{F}_{\mathrm{H}_{2} \mathrm{O}} \\
\mathrm{F}_{\mathrm{EtOH}}\end{array}$ & $\mathrm{L}$ \\
\hline 68 & & & 427 & $323,179,161,135$ & Caffeoyl malonylhexoside $(1)^{\mathrm{e}, \mathrm{f}}$ & $\mathrm{L}, \mathrm{S}^{\mathrm{a}}$ & $L, S^{a}$ & $\mathrm{~L}^{\mathrm{a}}, \mathrm{S}^{\mathrm{a}}$ & $\mathrm{L}, \mathrm{S}$ & $\mathrm{L}, \mathrm{S}$ & $\mathrm{L}^{\mathrm{a}}, \mathrm{S}^{\mathrm{a}}$ \\
\hline 69 & 12.9 & $\begin{array}{l}280 \\
310 \mathrm{sh}\end{array}$ & 697 & $\underline{535}, 371$ & p-Coumaroyl monotropein hexoside & $\mathrm{L}^{\mathrm{a}}$ & $\mathrm{F}_{\mathrm{H}_{2} \mathrm{O}}, \mathrm{F}_{\mathrm{EtOH}}$ & - & - & $\mathrm{F}_{\mathrm{H}_{2} \mathrm{O}}, \mathrm{F}_{\mathrm{EtOH}}$ & - \\
\hline 70 & 13.00 & $\begin{array}{l}285 \mathrm{sh}, \\
312\end{array}$ & 535 & $491, \underline{371}, 329,311,267,191,163$ & p-Coumaroyl monotropein (2) & $\mathrm{L}, \mathrm{S}$ & $\begin{array}{l}\mathrm{L}, \mathrm{S}, \mathrm{F}_{\mathrm{H}_{2} \mathrm{O}} \\
\mathrm{F}_{\mathrm{EtOH}}\end{array}$ & $\mathrm{L}, \mathrm{S}$ & $\mathrm{L}, \mathrm{S}$ & $\begin{array}{l}\mathrm{L}, \mathrm{S}, \mathrm{F}_{\mathrm{H}_{2} \mathrm{O}} \\
\mathrm{F}_{\mathrm{EtOH}}\end{array}$ & $\mathrm{L}^{\mathrm{a}}, \mathrm{S}$ \\
\hline 71 & 13.25 & 306 & 537 & $493, \underline{373}, 331,313,193,163$ & $\begin{array}{l}\text { p-Coumaroyl dihydromonotropein } \\
\text { (1) }\end{array}$ & $S^{a}$ & $\begin{array}{l}\mathrm{L}^{\mathrm{a}}, \mathrm{S}^{\mathrm{a}}, \mathrm{F}_{\mathrm{H}_{2} \mathrm{O}} \\
\mathrm{F}_{\mathrm{EtOH}}{ }^{\mathrm{a}}\end{array}$ & $\mathrm{L}^{\mathrm{a}}$ & $S^{a}$ & $\begin{array}{l}\mathrm{L}^{\mathrm{a}}, \mathrm{F}_{\mathrm{H}_{2} \mathrm{O}}{ }^{\mathrm{a}} \\
\mathrm{F}_{\mathrm{EtOH}}{ }^{2}\end{array}$ & - \\
\hline 72 & & 354 & 493 & $\underline{331}, 316$ & $3^{\prime}-O-$-Methylmyricetin hexoside ${ }^{\mathrm{d}}$ & - & $\mathrm{F}_{\mathrm{H}_{2} \mathrm{O}}, \mathrm{F}_{\mathrm{EtOH}}$ & - & - & $\mathrm{F}_{\mathrm{H}_{2} \mathrm{O}}, \mathrm{F}_{\mathrm{EtOH}}$ & - \\
\hline 73 & 13.40 & $\begin{array}{l}254 \\
352\end{array}$ & 433 & 301 & Quercetin pentoside (1) & $\mathrm{L}, \mathrm{S}$ & $\begin{array}{l}\mathrm{L}, \mathrm{S}, \mathrm{F}_{\mathrm{H}_{2} \mathrm{O}} \\
\mathrm{F}_{\mathrm{EtOH}}\end{array}$ & $\mathrm{L}, \mathrm{S}$ & $\mathrm{L}, \mathrm{S}$ & $\begin{array}{l}\mathrm{L}, \mathrm{S}, \mathrm{F}_{\mathrm{H}_{2} \mathrm{O}} \\
\mathrm{F}_{\mathrm{EtOH}}\end{array}$ & $\mathrm{L}, \mathrm{S}$ \\
\hline 74 & 13.55 & 324 & 381 & $191, \underline{179}, 161,135$ & Caffeoylquinic acid derivative (7) & $\mathrm{L}$ & $\begin{array}{l}\mathrm{L}, \mathrm{F}_{\mathrm{H}_{2} \mathrm{O}} \\
\mathrm{F}_{\mathrm{EtOH}}\end{array}$ & $\mathrm{L}^{\mathrm{a}}$ & $\mathrm{L}$ & $\mathrm{F}_{\mathrm{H}_{2} \mathrm{O}}, \mathrm{F}_{\mathrm{EtOH}}$ & - \\
\hline 75 & & & 577 & $559,451, \underline{425}, 407,289,287,245$ & B-type dimer (6) & $\mathrm{S}$ & $\begin{array}{l}\mathrm{L}, \mathrm{S}, \mathrm{F}_{\mathrm{H}_{2} \mathrm{O}} \\
\mathrm{F}_{\mathrm{EtOH}}{ }^{2}\end{array}$ & $\mathrm{~L}, \mathrm{~S}$ & $\mathrm{~L}, \mathrm{~S}$ & $\begin{array}{l}\mathrm{L}^{\mathrm{a}}, \mathrm{S}, \mathrm{F}_{\mathrm{H}_{2}} \mathrm{O}^{\mathrm{a}}, \\
\mathrm{F}_{\mathrm{EtOH}}\end{array}$ & $\mathrm{L}^{\mathrm{a}}, \mathrm{S}$ \\
\hline 76 & 13.75 & 312 & 537 & $493, \underline{373}, 331,313,193,163$ & $\begin{array}{l}\text { p-Coumaroyl dihydromonotropein } \\
\text { (2) }\end{array}$ & $\mathrm{L}, \mathrm{S}$ & $\begin{array}{l}\mathrm{L}, \mathrm{S}, \mathrm{F}_{\mathrm{H}_{2} \mathrm{O}} \\
\mathrm{F}_{\mathrm{EtOH}}\end{array}$ & $\mathrm{L}^{\mathrm{a}}, \mathrm{S}$ & $\mathrm{L}, \mathrm{S}$ & $\begin{array}{l}\mathrm{L}, \mathrm{S}, \mathrm{F}_{\mathrm{H}_{2} \mathrm{O}} \\
\mathrm{F}_{\mathrm{EtOH}}\end{array}$ & $\mathrm{S}$ \\
\hline 77 & 13.8 & & 433 & $\underline{301}, 271$ & Quercetin pentoside (2) & - & $\mathrm{F}_{\mathrm{H}_{2} \mathrm{O}}, \mathrm{F}_{\mathrm{EtOH}}$ & - & - & $\mathrm{F}_{\mathrm{H}_{2} \mathrm{O}}, \mathrm{F}_{\mathrm{EtOH}}{ }^{\mathrm{a}}$ & - \\
\hline 78 & 13.95 & & 409 & $325,307,217, \underline{187}, 163,159,145$ & $p$-Coumaroyl diacetylhexoside $(1)^{\mathrm{e}}$ & $\mathrm{L}, \mathrm{S}$ & $\begin{array}{l}\mathrm{L}, \mathrm{S}, \mathrm{F}_{\mathrm{H}_{2} \mathrm{O}} \\
\mathrm{F}_{\mathrm{EtOH}}\end{array}$ & $\mathrm{L}, \mathrm{S}$ & $\mathrm{L}, \mathrm{S}$ & $\begin{array}{l}\mathrm{L}, \mathrm{S}, \mathrm{F}_{\mathrm{H}_{2} \mathrm{O}} \\
\mathrm{F}_{\mathrm{EtOH}}\end{array}$ & $\mathrm{L}, \mathrm{S}$ \\
\hline 79 & & & 461 & 285 & Kaempferol hexuronide ${ }^{\mathrm{c}, \mathrm{e}, \mathrm{f}}$ & $\mathrm{L}$ & $\begin{array}{l}\mathrm{L}, \mathrm{F}_{\mathrm{H}_{2} \mathrm{O}} \\
\mathrm{F}_{\mathrm{EtOH}}\end{array}$ & $\mathrm{L}$ & $\mathrm{L}$ & $\begin{array}{l}\mathrm{L}, \mathrm{F}_{\mathrm{H}_{2} \mathrm{O}} \\
\mathrm{F}_{\mathrm{EtOH}}\end{array}$ & $\mathrm{L}$ \\
\hline 80 & 14.05 & 278 & 865 & $739,713, \underline{695}, 577,561,543,525$, & B-type trimer (5) & $\mathrm{S}$ & $\mathrm{S}$ & $S^{a}$ & $S^{a}$ & $\mathrm{~S}$ & $S^{a}$ \\
\hline
\end{tabular}
407,285 
Version définitive du manuscrit publiée dans / Final version of the manuscript published in :

Food Chemistry (2016), Vol. 213, p. 58-68, DOI: 10.1016/j.foodchem.2016.06.042

Journal homepage : www.elsevier.com/locate/foodchem

Table 1 (continued)

\begin{tabular}{|c|c|c|c|c|c|c|c|c|c|c|c|}
\hline \multirow[t]{2}{*}{ No. } & \multirow{2}{*}{$\begin{array}{l}\mathrm{t}_{\mathrm{R}} \\
(\mathrm{min})\end{array}$} & \multirow{2}{*}{$\begin{array}{l}\lambda_{\max } \\
(\mathrm{nm})\end{array}$} & \multirow{2}{*}{$\begin{array}{l}{[\mathrm{M}-\mathrm{H}]^{-}} \\
(m / z)\end{array}$} & \multirow[t]{2}{*}{$\mathrm{MS}^{2}$ fragments $(m / z)$} & \multirow[t]{2}{*}{ Proposed structure } & \multicolumn{3}{|l|}{2013} & \multicolumn{3}{|l|}{2014} \\
\hline & & & & & & May & July & Sept. & May & July & Sept. \\
\hline 81 & 14.15 & $\begin{array}{l}254 \\
352\end{array}$ & 447 & 301 & Quercetin-3-O-rhamnoside (std) $)^{\mathrm{c}, \mathrm{e}, \mathrm{f}}$ & $\mathrm{L}, \mathrm{S}$ & $\begin{array}{l}\mathrm{L}, \mathrm{S}, \mathrm{F}_{\mathrm{H}_{2} \mathrm{O}} \\
\mathrm{F}_{\mathrm{EtOH}}\end{array}$ & $\mathrm{L}, \mathrm{S}$ & $\mathrm{L}, \mathrm{S}$ & $\begin{array}{l}\mathrm{L}, \mathrm{S}, \mathrm{F}_{\mathrm{H}_{2} \mathrm{O}} \\
\mathrm{F}_{\mathrm{EtOH}}\end{array}$ & $\mathrm{L}, \mathrm{S}$ \\
\hline 82 & 14.20 & & 535 & $\underline{491}, 371,355,329,311,191,163$ & p-Coumaroyl monotropein (3) & $\begin{array}{l}\mathrm{L}^{\mathrm{a}} \\
\mathrm{S}^{\mathrm{a}}\end{array}$ & $\begin{array}{l}\mathrm{S}^{\mathrm{a}}, \mathrm{F}_{\mathrm{H}_{2} \mathrm{O}} \\
\mathrm{F}_{\mathrm{EtOH}}\end{array}$ & $\mathrm{L}^{\mathrm{a}}, \mathrm{S}^{\mathrm{a}}$ & $\mathrm{L}^{\mathrm{a}}, \mathrm{S}^{\mathrm{a}}$ & $\begin{array}{l}\mathrm{L}^{\mathrm{a}}, \mathrm{S}^{\mathrm{a}} \mathrm{F}_{\mathrm{H}_{2} \mathrm{O}} \\
\mathrm{F}_{\mathrm{EtOH}}\end{array}$ & - \\
\hline 83 & 14.25 & & 411 & $307,163, \underline{145}, 119,117$ & $\begin{array}{l}\text { p-Coumaroyl malonylhexoside } \\
(1)^{\text {e,f,g }}\end{array}$ & $\mathrm{L}, \mathrm{S}$ & $\begin{array}{l}\mathrm{L}, \mathrm{S}, \mathrm{F}_{\mathrm{H}_{2} \mathrm{O}} \\
\mathrm{F}_{\mathrm{EtOH}}\end{array}$ & $\mathrm{L}, \mathrm{S}$ & $\mathrm{L}, \mathrm{S}$ & $\begin{array}{l}\mathrm{L}, \mathrm{S}, \mathrm{F}_{\mathrm{H}_{2} \mathrm{O}} \\
\mathrm{F}_{\mathrm{EtOH}}\end{array}$ & $\mathrm{L}, \mathrm{S}$ \\
\hline 84 & 14.35 & $\begin{array}{l}285 \mathrm{sh}, \\
312\end{array}$ & 535 & $491,373,355, \underline{329}, 311,201,163$ & p-Coumaroyl monotropein (4) & $\mathrm{L}, \mathrm{S}^{\mathrm{a}}$ & $\begin{array}{l}\mathrm{S}, \mathrm{F}_{\mathrm{H}_{2} \mathrm{O}} \\
\mathrm{F}_{\mathrm{EtOH}}\end{array}$ & $\mathrm{L}^{\mathrm{a}}, \mathrm{S}$ & $S^{a}$ & $\begin{array}{l}\mathrm{S}^{\mathrm{a}}, \mathrm{F}_{\mathrm{H}_{2} \mathrm{O}} \\
\mathrm{F}_{\mathrm{EtOH}}\end{array}$ & - \\
\hline 85 & 14.50 & & 451 & $\underline{341}, 217$ & Cinchonain I (4) & - & $\mathrm{F}_{\mathrm{EtOH}}^{\mathrm{a}}$ & - & - & $\begin{array}{l}\mathrm{L}, \mathrm{F}_{\mathrm{H}_{2} \mathrm{O}} \mathrm{a} \\
\mathrm{F}_{\mathrm{EtOH}}\end{array}$ & $\mathrm{L}$ \\
\hline 86 & 14.55 & $\begin{array}{l}254 \\
350\end{array}$ & 579 & $475,447,429,355, \underline{300}, 271$ & Quercetin pentosyldeoxyhexoside & $S$ & $\mathrm{~S}$ & $\mathrm{~S}$ & S & $\mathrm{S}$ & $\mathrm{S}$ \\
\hline 87 & 14.60 & $\begin{array}{l}290 \\
324\end{array}$ & 427 & $265, \underline{179}, \underline{161}, 135$ & Caffeoyl malonylhexoside (2) & $\mathrm{L}, \mathrm{S}$ & $\begin{array}{l}\mathrm{L}, \mathrm{S}, \mathrm{F}_{\mathrm{H}_{2} \mathrm{O}} \\
\mathrm{F}_{\mathrm{EtOH}}\end{array}$ & $\mathrm{L}, \mathrm{S}$ & $\mathrm{L}, \mathrm{S}$ & $\begin{array}{l}\mathrm{L}, \mathrm{S}, \mathrm{F}_{\mathrm{H}_{2} \mathrm{O}} \\
\mathrm{F}_{\mathrm{EtOH}}\end{array}$ & $\mathrm{L}, \mathrm{S}$ \\
\hline 88 & & & 573 & $\underline{411}, 393,249,163$ & p-Coumaroyl malonyldihexoside (1) & $\mathrm{L}^{\mathrm{a}}, \mathrm{S}$ & $\mathrm{L}, \mathrm{S}$ & $\mathrm{L}, \mathrm{S}$ & $\mathrm{L}^{\mathrm{a}}, \mathrm{S}$ & $\mathrm{L}^{\mathrm{a}}, \mathrm{S}$ & $\mathrm{L}^{\mathrm{a}}, \mathrm{S}^{\mathrm{a}}$ \\
\hline 89 & 14.65 & & 507 & $\begin{array}{l}387, \underline{343}, 329,301,273,179,163 \text {, } \\
151\end{array}$ & $p$-Coumaric acid derivative & - & $\mathrm{F}_{\mathrm{H}_{2} \mathrm{O}}, \mathrm{F}_{\mathrm{EtOH}}$ & - & - & $\mathrm{F}_{\mathrm{H}_{2} \mathrm{O}}, \mathrm{F}_{\mathrm{EtOH}}$ & - \\
\hline 90 & 14.70 & & 521 & $\underline{345}, 329$ & Syringetin hexuronic acid ${ }^{\mathrm{d}}$ & - & $\mathrm{F}_{\mathrm{H}_{2} \mathrm{O}}, \mathrm{F}_{\mathrm{EtOH}}$ & - & - & $\mathrm{F}_{\mathrm{H}_{2} \mathrm{O}}, \mathrm{F}_{\mathrm{EtOH}}$ & - \\
\hline 91 & 15.0 & & 435 & 273 & Phloretin-2-0-hexoside & - & $\begin{array}{l}\mathrm{F}_{\mathrm{H}_{2} \mathrm{O}} \\
\mathrm{F}_{\mathrm{EtOH}}\end{array}$ & - & - & $\mathrm{F}_{\mathrm{H}_{2} \mathrm{O}}, \mathrm{F}_{\mathrm{EtOH}}$ & - \\
\hline 92 & 15.15 & $\begin{array}{l}290 \mathrm{sh}, \\
306\end{array}$ & 409 & $325,307,217, \underline{187}, 163,159,145$ & p-Coumaroyl diacetylhexoside (2) & $\mathrm{L}, \mathrm{S}$ & $\begin{array}{l}\mathrm{L}, \mathrm{S}, \mathrm{F}_{\mathrm{H}_{2} \mathrm{O}} \\
\mathrm{F}_{\mathrm{EtOH}}\end{array}$ & $\mathrm{L}, \mathrm{S}$ & $\mathrm{L}, \mathrm{S}$ & $\begin{array}{l}\mathrm{L}, \mathrm{S}, \mathrm{F}_{\mathrm{H}_{2} \mathrm{O}} \\
\mathrm{F}_{\mathrm{EtOH}}\end{array}$ & $\mathrm{L}, \mathrm{S}$ \\
\hline 93 & 15.25 & & 573 & $411,393,163$ & p-Coumaroyl malonyldihexoside (2) & S & S & S & S & $\mathrm{S}$ & $\mathrm{S}$ \\
\hline 94 & 15.3 & $\begin{array}{l}286 \\
306\end{array}$ & 411 & $307, \underline{163}, \underline{145}, 119$ & p-Coumaroyl malonylhexoside (2) & $\mathrm{L}, \mathrm{S}$ & $\begin{array}{l}\mathrm{L}, \mathrm{S}, \mathrm{F}_{\mathrm{H}_{2} \mathrm{O}} \\
\mathrm{F}_{\mathrm{EtOH}}\end{array}$ & $\mathrm{L}, \mathrm{S}$ & $\mathrm{L}, \mathrm{S}$ & $\begin{array}{l}\mathrm{L}, \mathrm{S}, \mathrm{F}_{\mathrm{H}_{2} \mathrm{O}} \\
\mathrm{F}_{\mathrm{EtOH}}\end{array}$ & $\mathrm{L}, \mathrm{S}$ \\
\hline 95 & 15.35 & $\begin{array}{l}280 \\
306\end{array}$ & 543 & $\underline{411}, 163$ & $\begin{array}{l}\text { p-Coumaroyl } \\
\text { malonylpentosylhexoside }\end{array}$ & $S^{a}$ & $\mathrm{~S}$ & $\mathrm{~S}$ & $\mathrm{~S}$ & $\mathrm{~S}$ & $\mathrm{~S}$ \\
\hline 96 & 15.55 & $\begin{array}{l}285 \mathrm{sh}, \\
310\end{array}$ & 411 & $249, \underline{163}, 145,119$ & p-Coumaroyl malonylhexoside (3) & $\mathrm{L}, \mathrm{S}$ & $\begin{array}{l}\mathrm{L}, \mathrm{S}, \mathrm{F}_{\mathrm{H}_{2} \mathrm{O}} \\
\mathrm{F}_{\mathrm{EtOH}}\end{array}$ & $\mathrm{L}, \mathrm{S}$ & $\mathrm{L}, \mathrm{S}$ & $\begin{array}{l}\mathrm{L}, \mathrm{S}, \mathrm{F}_{\mathrm{H}_{2} \mathrm{O}} \\
\mathrm{F}_{\mathrm{EtOH}}\end{array}$ & $\mathrm{L}, \mathrm{S}$ \\
\hline 97 & 15.65 & $\begin{array}{l}286 \\
304\end{array}$ & 411 & $249, \underline{163}, 145,119$ & p-Coumaroyl malonylhexoside (4) & $\mathrm{L}, \mathrm{S}$ & $\begin{array}{l}\mathrm{L}, \mathrm{S}, \mathrm{F}_{\mathrm{H}_{2} \mathrm{O}} \\
\mathrm{F}_{\mathrm{EtOH}}\end{array}$ & $\mathrm{L}, \mathrm{S}$ & $\mathrm{L}, \mathrm{S}$ & $\begin{array}{l}\mathrm{L}, \mathrm{S}, \mathrm{F}_{\mathrm{H}_{2} \mathrm{O}} \\
\mathrm{F}_{\mathrm{EtOH}}\end{array}$ & $\mathrm{L}, \mathrm{S}$ \\
\hline 98 & 15.8 & $\begin{array}{l}290 \text { sh, } \\
312\end{array}$ & 451 & $341,307,229, \underline{187}, 163$ & $p$-Coumaroyl triacetylhexoside $(1)^{c, e}$ & $\mathrm{~L}$ & - & - & $\mathrm{L}$ & - & - \\
\hline 99 & & 280 & 451 & $\underline{341}, 217$ & Cinchonain I (5) & - & $\begin{array}{l}\mathrm{L}, \mathrm{S}, \mathrm{F}_{\mathrm{H}_{2} \mathrm{O}} \\
\mathrm{F}_{\mathrm{EtOH}}\end{array}$ & $\mathrm{L}, \mathrm{S}$ & $\mathrm{S}$ & $\begin{array}{l}\mathrm{L}, \mathrm{S}, \mathrm{F}_{\mathrm{H}_{2} \mathrm{O}} \\
\mathrm{F}_{\mathrm{EtOH}}\end{array}$ & $\mathrm{L}, \mathrm{S}$ \\
\hline 100 & 15.9 & $\begin{array}{l}254 \\
350\end{array}$ & 591 & $529,489, \underline{447}, 301$ & $\begin{array}{l}\text { Quercetin-3-O-(4"-HMG)- } \alpha- \\
\text { rhamnoside }{ }^{\mathrm{e}, \mathrm{f}, \mathrm{j}}\end{array}$ & $\mathrm{L}, \mathrm{S}$ & $\begin{array}{l}\mathrm{L}, \mathrm{S}, \mathrm{F}_{\mathrm{H}_{2}} \mathrm{O} \\
\mathrm{F}_{\mathrm{EtOH}}\end{array}$ & $\mathrm{L}, \mathrm{S}$ & $\mathrm{L}^{\mathrm{a}}, \mathrm{S}$ & $\begin{array}{l}\mathrm{L}, \mathrm{S}, \mathrm{F}_{\mathrm{H}_{2} \mathrm{O}} \\
\mathrm{F}_{\mathrm{EtOH}}\end{array}$ & $\mathrm{L}, \mathrm{S}$ \\
\hline 101 & & & 425 & $\underline{179}, 135$ & Caffeoyl derivative & - & $\begin{array}{l}\mathrm{F}_{\mathrm{H}_{2} \mathrm{O}}{ }^{\mathrm{a}} \\
\mathrm{F}_{\mathrm{EtOH}}\end{array}$ & - & - & $\mathrm{F}_{\mathrm{H}_{2} \mathrm{O}}, \mathrm{F}_{\mathrm{EtOH}}$ & - \\
\hline 102 & 15.95 & $\begin{array}{l}290 \text { sh, } \\
312\end{array}$ & 451 & $\begin{array}{l}367,349,307,245,203, \underline{187}, 159, \\
145\end{array}$ & p-Coumaroyl triacetylhexoside (2) & $\mathrm{L}$ & - & - & $\mathrm{L}$ & - & - \\
\hline 103 & & & 451 & $\underline{341}, 299$ & Cinchonain I (6) & $S^{a}$ & $\mathrm{~L}, \mathrm{~S}^{\mathrm{a}}$ & $\mathrm{L}, \mathrm{S}^{\mathrm{a}}$ & $S^{\mathrm{a}}$ & $\mathrm{L}, \mathrm{S}^{\mathrm{a}}$ & $L, S^{a}$ \\
\hline 104 & & $\begin{array}{l}284 \\
314\end{array}$ & 445 & $\underline{179}, 135$ & Caffeoyl derivative ${ }^{g}$ & - & $\mathrm{F}_{\mathrm{H}_{2} \mathrm{O}}, \mathrm{F}_{\mathrm{EtOH}}$ & - & - & $\mathrm{F}_{\mathrm{H}_{2} \mathrm{O}}, \mathrm{F}_{\mathrm{EtOH}}$ & - \\
\hline 105 & 16.15 & $\begin{array}{l}290 \mathrm{sh}, \\
312\end{array}$ & 451 & $\begin{array}{l}367,349,307,245,203, \underline{187}, 159, \\
145\end{array}$ & p-Coumaroyl triacetylhexoside (3) & $\mathrm{L}$ & $\mathrm{L}^{\mathrm{a}}$ & $\mathrm{L}^{\mathrm{a}}$ & $\mathrm{L}$ & $\mathrm{L}$ & $\mathrm{L}^{\mathrm{a}}$ \\
\hline 106 & 16.6 & $\begin{array}{l}286 \\
310\end{array}$ & 249 & $163, \underline{145}$ & Malonyl p-coumaric acid ${ }^{e}$ & $\mathrm{~S}$ & $\mathrm{~S}$ & $\mathrm{~S}$ & $S$ & $\mathrm{~S}$ & $\mathrm{~S}$ \\
\hline
\end{tabular}

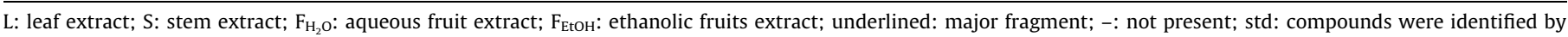
comparison with standards.

a Not fragmented.

b Doubly-charged ion. Compounds in bold are newly described or identified.

c Hokkanen, Mattila, Jaakola, Pirttilä, and Tolonen (2009).

d Lätti, Jaakola, Riihinen, and Kainulainen (2010).

e Ieri, Martini, Innocenti, \& Mulinacci (2013).

f Liu, Lindstedt, Markkinen, Sinkkonen, Suomela, \& Yang (2014).

g Mikulic-Petkovsek, Schmitzer, Slatnar, Stampar, \& Veberic (2015).

h Chanforan, Loonis, Mora, Caris-Veyrat, \& Dufour (2012).

i Mane, Loonis, Juhel, Dufour, \& Malien-Aubert (2011).

j $\mathrm{HMG}=3$-hydroxy-3-methylglutaryl.

two $p$-coumaroyl malonyldihexosides and one p-coumaroyl malonylhexosylpentoside, the last two molecules being mostly present in stems. p-Coumaroyl malonylhexosides $(83,94,96$ and 97; $\mathrm{m} / z$ 411) display fragment ions at $\mathrm{m} / \mathrm{z} 307$ (loss of malonic acid) or 249 (loss of hexose), 163,145, and 119. Newly identified $p$ coumaroyl malonyldihexosides (88 and 93) and $p$-coumaroyl malonylpentosylhexoside (95) have parent ions at $\mathrm{m} / \mathrm{z} 573$ and
543 , respectively, and a common major fragment ion at $m / z 411$. When accessible, absorption spectra are showing dual $\lambda_{\max }$ at $c a$. 286 and $310 \mathrm{~nm}$ as found for the last compound to be eluted (106). This apolar compound has a parent ion at $\mathrm{m} / \mathrm{z} 249$ and its structure could be attributed to 4-O-malonyl $p$-coumaric acid. As a matter of fact, this large family of glycosides linked to both p-coumaric acid and malonic acid may encompass three main 
Version définitive du manuscrit publiée dans / Final version of the manuscript published in :

Food Chemistry (2016), Vol. 213, p. 58-68, DOI: 10.1016/j.foodchem.2016.06.042

Journal homepage : www.elsevier.com/locate/foodchem

Table 2

Phenolic composition in bilberry leaves, stems and fruits at three different periods of vegetation and for two different years.

\begin{tabular}{|c|c|c|c|c|c|c|c|c|c|}
\hline $\begin{array}{l}\text { Extract/ } \\
\text { Period of } \\
\text { vegetation }\end{array}$ & $\begin{array}{l}\text { Caffeic acid } \\
\text { derivatives } \\
(\mathrm{mg} / \mathrm{g} \mathrm{DE})\end{array}$ & $\begin{array}{l}\text { Coumaric acid } \\
\text { derivatives } \\
(\mathrm{mg} / \mathrm{g} \mathrm{DE})\end{array}$ & $\begin{array}{l}\text { Flavonol } \\
\text { glycosides } \\
(\mathrm{mg} / \mathrm{g} \mathrm{DE})\end{array}$ & $\begin{array}{l}\text { Flavanol } \\
\text { monomers } \\
(\mathrm{mg} / \mathrm{g} \mathrm{DE})\end{array}$ & $\begin{array}{l}\text { Flavanol } \\
\text { oligomers } \\
(\mathrm{mg} / \mathrm{g} \mathrm{DE})\end{array}$ & $\begin{array}{l}\text { Anthocyanins } \\
\text { (mg/g DE) }\end{array}$ & $\begin{array}{l}\text { Sum of phenolic } \\
\text { compounds } \\
\text { (mg/g DE) }\end{array}$ & $\begin{array}{l}\text { Total Phenolic } \\
\text { Content (mg } \\
\text { GAE/g DE) }\end{array}$ & $\begin{array}{l}\text { Total Phenolic } \\
\text { Content (mg } \\
\text { GAE/g DM) }\end{array}$ \\
\hline \multicolumn{10}{|l|}{ Leaves } \\
\hline May 2013 & $65.2 \pm 5.6(\mathrm{~A})$ & $21.6 \pm 2.3(\mathrm{~A})$ & $10.6 \pm 0.5(\mathrm{~A})$ & - & - & - & $97.4 \pm 7.9(\mathrm{~A})$ & - & $54.7 \pm 3.9(\mathrm{~A})$ \\
\hline May 2014 & $124.6 \pm 3.5(a)^{*}$ & $35.8 \pm 1.4(a)^{*}$ & $10.4 \pm 3.7(a)$ & - & - & - & $170.8 \pm 4.4(a)^{*}$ & $118.7 \pm 2.4(a)$ & $75.1 \pm 1.6(a)^{*}$ \\
\hline July 2013 & $98.0 \pm 10.6(\mathrm{~B})$ & $8.83 \pm 0.78(\mathrm{~B})$ & $15.8 \pm 3.2(\mathrm{~A}, \mathrm{~B})$ & $1.12 \pm 0.22(\mathrm{~A})$ & $1.10 \pm 0.14(\mathrm{~A})^{b}$ & - & $124.9 \pm 14.4(\mathrm{~B})$ & - & $105.7 \pm 6.0(\mathrm{~B})$ \\
\hline July 2014 & $100.5 \pm 0.6(b)$ & $10.2 \pm 0.0(b)^{*}$ & $22.5 \pm 0.5(b)^{*}$ & $1.36 \pm 0.15(a)$ & $1.33 \pm 0.34(a)^{b}$ & - & $135.9 \pm 1.9(b)$ & $166.1 \pm 4.4(b)$ & $106.9 \pm 2.9(b)$ \\
\hline September 2013 & $72.1 \pm 4.4(\mathrm{~A})$ & $7.48 \pm 0.25(\mathrm{~B})$ & $17.9 \pm 2.0(\mathrm{~B})$ & $0.53 \pm 0.21(B)$ & $1.87 \pm 0.08(\mathrm{~B})^{b}$ & - & $99.9 \pm 6.7(\mathrm{~A}, \mathrm{~B})$ & - & $102.4 \pm 5.3(\mathrm{~B})$ \\
\hline September 2014 & $72.3 \pm 0.7(c)$ & $7.91 \pm 0.21(c)$ & $14.0 \pm 0.3(a)^{*}$ & $1.01 \pm 0.28(a)^{*}$ & $1.37 \pm 0.35(a)^{b}$ & - & $96.6 \pm 0.6(c)$ & $142.9 \pm 19.2(a, b)$ & $87.1 \pm 11.7(a)$ \\
\hline \multicolumn{10}{|l|}{ Stems } \\
\hline May 2013 & $7.16 \pm 0.18(\mathrm{~A})$ & $9.54 \pm 0.29(\mathrm{~A})$ & $11.5 \pm 1.4(\mathrm{~A})$ & $4.39 \pm 1.49(\mathrm{~A})$ & $40.0 \pm 2.9(\mathrm{~A})$ & - & $71.0 \pm 5.9(\mathrm{~A})$ & - & $72.4 \pm 14.4(\mathrm{~A})$ \\
\hline May 2014 & $9.79 \pm 0.53(a)^{*}$ & $11.5 \pm 0.2(a)^{*}$ & $14.5 \pm 0.1(a)^{*}$ & $7.24 \pm 0.77(a)^{*}$ & $49.7 \pm 0.7(a)^{*}$ & - & $92.7 \pm 1.2(a)^{*}$ & $136.6 \pm 4.1(a)$ & $73.1 \pm 2.2(a)$ \\
\hline July 2013 & $7.58 \pm 0.33(\mathrm{~A})$ & $11.5 \pm 0.6(B)$ & $9.63 \pm 0.89(\mathrm{~A}, \mathrm{~B})$ & $6.31 \pm 1.63(\mathrm{~A})$ & $49.1 \pm 6.2(\mathrm{~A})$ & - & $79.3 \pm 1.7(\mathrm{~A})$ & - & $78.8 \pm 9.3(\mathrm{~A})$ \\
\hline July 2014 & $10.2 \pm 0.1(a)^{*}$ & $13.3 \pm 0.3(b)^{*}$ & $16.4 \pm 1.0(b)^{*}$ & $10.6 \pm 0.80(b)^{*}$ & $71.3 \pm 5.3(b)^{*}$ & - & $121.8 \pm 4.8(b)^{*}$ & $174.3 \pm 2.8(b)$ & $98.7 \pm 4.6(b)^{*}$ \\
\hline September 2013 & $6.90 \pm 0.36(\mathrm{~A})$ & $9.26 \pm 0.29(\mathrm{~A})$ & $8.71 \pm 0.38(\mathrm{~B})$ & $10.9 \pm 4.0(\mathrm{~A})$ & $48.7 \pm 2.0(\mathrm{~A})$ & - & $80.8 \pm 0.3(\mathrm{~A})$ & - & $81.2 \pm 4.8(\mathrm{~A})$ \\
\hline September 2014 & $5.87 \pm 0.05(b)^{*}$ & $11.7 \pm 0.2(a)^{*}$ & $9.22 \pm 0.38(c)$ & $11.9 \pm 1.7(b)$ & $57.1 \pm 0.4(a)^{*}$ & - & $95.8 \pm 1.9(a)^{*}$ & $140.0 \pm 18.8(a)$ & $81.8 \pm 11.0(a)$ \\
\hline \multicolumn{10}{|c|}{ Fruits with $\mathrm{H}_{2} \mathrm{O}$} \\
\hline July 2013 & $2.57 \pm 0.42$ & $1.12 \pm 0.08$ & $0.96 \pm 0.16$ & - & - & $22.3 \pm 1.0$ & $26.9 \pm 1.7$ & - & $31.8 \pm 1.2$ \\
\hline July 2014 & $2.44 \pm 0.25$ & $1.45 \pm 0.14^{*}$ & $0.99 \pm \pm 0.16$ & $0.11 \pm 0.06^{a}$ & - & $29.6 \pm 5.8$ & $34.7 \pm 5.6$ & $38.6 \pm 2.2$ & $30.5 \pm 1.7$ \\
\hline \multicolumn{10}{|c|}{ Fruits with EtOH 55\% } \\
\hline July 2013 & $3.54 \pm 0.43$ & $1.49 \pm 0.14$ & $1.39 \pm 0.23$ & $0.13 \pm 0.05^{a}$ & - & $34.5 \pm 10.3$ & $41.1 \pm 11.1$ & - & $41.9 \pm 1.7$ \\
\hline July 2014 & $2.36 \pm 0.07^{*}$ & $1.47 \pm 0.07$ & $0.94 \pm 0.03^{*}$ & $0.12 \pm 0.07^{a}$ & - & $25.7 \pm 4.0$ & $30.6 \pm 4.1$ & $33.1 \pm 0.9$ & $34.7 \pm 1.0^{*}$ \\
\hline
\end{tabular}

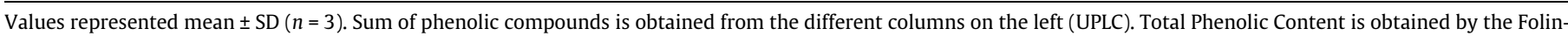

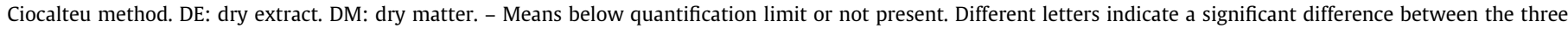
periods of vegetation at $p<0.05$; capital and small letters are used to compare the samples from 2013 and 2014 , respectively.

* Means a significant difference between the two years $(p<0.05)$.

a Flavanol monomers in fruits contain only a cinchonain I isomer.

b Flavanol oligomers contain B-type and A-type oligomers in stems, only cinchonains I + II in leaves.

structures. Malonic acid could be grafted on the 4-OH group of $p$ coumaric acid or on the hexose unit when $p$-coumaric acid can be either etherified or esterified by the hexose unit, in agreement with the earlier identification of $p$-coumaric acid-4-O-hexosides and $p$-coumaroylhexosides, respectively. Compounds with parent ions at $\mathrm{m} / \mathrm{z}$ 409, 411 and 451 were already found in bud and leaf extracts of bilberry by Ieri et al. (2013), Liu et al. (2014), and Mikulic-Petkovsek et al. (2015), although they were only named as $p$-coumaroyl derivatives.

Several iridoid glycosides acylated by $p$-coumaric acid $(m / z 535)$ were identified in all the morphological parts and assigned as $p$ coumaroyl monotropeins $(63,70,82$ and 84). $p$-Coumaroyl dihydromonotropein isomers ( $\mathbf{7 1}$ and $\mathbf{7 6}$ ) were newly identified in bilberry similarly to a compound with a parent ion at $m / z 697$, which was attributed to $p$-coumaroyl monotropein hexoside $(\mathbf{6 9})$ through major fragments at $\mathrm{m} / \mathrm{z} 535$ ( $p$-coumaroyl monotropein) and $\mathrm{m} / \mathrm{z}$ 371 (subsequent loss of coumaric acid). The latter was found in trace amounts in fruits and leaves.

\subsubsection{Flavonol glycosides}

In stems and leaves, quercetin glycosides were present in considerable amounts from May to September (Table 2), whereas in fruits from July they appeared in lower concentrations. Quercetin-3-O-galactoside (62), quercetin-3-O-glucoside (67), quercetin hexuronides $(\mathbf{6 1}, \mathbf{6 4}$ and $\mathbf{6 6})$, quercetin pentosides (73 and $\mathbf{7 7})$, and a quercetin rhamnoside (81) were observed (Table 1$)$. The berry characteristic quercetin-3-O-(4"-(3-hydroxy-3-methyl glutaryl))- $\alpha$-rhamnoside (100) was identified in all the morphological parts. This compound is newly described in bilberry fruit while it was evidenced in bilberry buds and leaves by Hokkanen et al. (2009), and Ieri et al. (2013). It was quantified in leaves in July and September (4th most abundant flavonol) and in stems from all seasons.

The last two quercetin glycosides presented similar fragmentation pathways with the loss of 132 and 150 amu characteristic of a pentose unit. Quercetin pentosylhexoside $(\mathbf{5 6}, \mathrm{m} / \mathrm{z} 595)$ and quer- cetin pentosyldeoxyhexoside $(\mathbf{8 6}, \mathrm{m} / \mathrm{z} 579)$ were newly identified in stems.

Finally, a dihydrochalcone was newly identified in bilberry fruit and assigned as phloretin hexoside (91), displaying a parent ion at $\mathrm{m} / \mathrm{z} 435$ and a fragment ion at $\mathrm{m} / \mathrm{z} 273$ (loss of hexose).

\subsubsection{Flavanols}

In stems, flavanols were present from May to September (Table 1) although they tended to be more abundant in July and September (Table 2). Epicatechin or catechin-based oligomeric flavanols encompass a large variety with various B-type dimeric (16, $25,32,42,49$ and 75$)$, trimeric $(28,33,46,58$ and 80$)$, and tetrameric forms (40, 43 and $\mathbf{5 5}$ ). A-type dimers ( 47 and $\mathbf{5 2}$ ) and trimers (12, 41 and 45) were also present, the latter resulting from an intramolecular two-electron oxidation of the B-type corresponding structures. Additionally, (-)-epigallocatechin (15) and (-)epicatechin (34) were present in quantifiable amounts, whereas (+)-gallocatechin (1) was only detected by mass spectrometry. (Epi)gallocatechin was further identified in three mixed B-type dimers with (epi)catechin (11, 19 and 48), a mixed B-type trimer (36) and a mixed A-type trimer (39). Coupling between caffeic acid and monomeric or dimeric flavanols led to five cinchonain I isomers (26, 37, 60, 99 and 103) and two cinchonain II isomers (38 and 59), respectively. Two main fragmentation pathways were observed for cinchonains I with isomers giving major fragment ions at $m / z 289$ and 245 and others at $m / z 341$ and 217. None of them were quantified, being either minor compounds in coeluted peaks or present below the limit of quantification.

In leaves, eight B-type dimers (25, 32 and $\mathbf{7 5}$ ), trimers (33 and 46), and tetramers (40, 43 and $\mathbf{5 5}$ ) and one A-type dimer (47) were identified but not quantified, when only one B-type dimer (75) was identified in fruits. (-)-Epicatechin (34) was only present in leaves from July and September, representing less than $1 \%$ of the phenolic pool (Table 3). Lastly, cinchonains I (60, 85, 99 and 103) and II (59) were identified in leaves when only two cinchonain I (85 and $\mathbf{9 9})$ were present in fruits. Cinchonains were only quantified in leaves 
Version définitive du manuscrit publiée dans / Final version of the manuscript published in :

Food Chemistry (2016), Vol. 213, p. 58-68, DOI: 10.1016/j.foodchem.2016.06.042

Journal homepage : www.elsevier.com/locate/foodchem

Table 3

Relative content of major phenolic compounds in bilberry leaves and stems at three different periods of vegetation and for two different years.

\begin{tabular}{|c|c|c|c|c|c|c|c|}
\hline \multirow[t]{3}{*}{ Morphological parts } & \multirow[t]{3}{*}{ Major phenolic compounds ${ }^{a}$} & \multicolumn{6}{|c|}{ Relative content $(\%)^{\mathrm{b}}$} \\
\hline & & \multicolumn{3}{|l|}{2013} & \multicolumn{3}{|l|}{2014} \\
\hline & & May & July & Sept. & May & July & Sept. \\
\hline \multirow[t]{12}{*}{ Leaf extracts } & 5-O-Caffeoylquinic-acid (18) & 55.6 & 74.6 & 68.3 & 67.9 & 70.0 & 70.3 \\
\hline & 5-O-Caffeoylquinic acid (cis) (31) & 0.8 & 1.3 & 1.1 & 0.7 & 1.2 & 1.6 \\
\hline & Caffeoyl malonylhexoside (87) & 1.5 & 0.9 & 1.2 & 1.1 & 0.9 & 1.2 \\
\hline & Quercetin-3-O-galactoside (62) & 1.4 & 4.6 & 7.8 & 1.1 & 3.3 & 1.9 \\
\hline & Quercetin hexuronide (64) & 8.9 & 6.2 & 8.1 & 4.8 & 11.5 & 11.7 \\
\hline & Quercetin pentoside (73) & 0.5 & 1.4 & 1.7 & 0.2 & 1.2 & 0.6 \\
\hline & 5-p-Coumaroylquinic acid (30) & 2.0 & 0.6 & 0.4 & 2.0 & 0.4 & 0.4 \\
\hline & p-Coumaroyl monotropein (70) & 5.1 & 0.5 & 0.3 & 4.5 & 0.5 & 0.1 \\
\hline & p-Coumaroyl diacetylhexoside (78) & 5.3 & 1.5 & 1.4 & 5.6 & 1.6 & 1.0 \\
\hline & p-Coumaroyl malonylhexoside (83) & 1.2 & 1.0 & 1.3 & 0.8 & 1.4 & 2.4 \\
\hline & p-Coumaroyl malonylhexoside (96) & 2.6 & 1.8 & 2.3 & 2.8 & 1.9 & 2.4 \\
\hline & $(-)$-Epicatechin $(34)$ & - & 0.9 & 0.5 & - & 1.0 & 1.0 \\
\hline \multirow[t]{10}{*}{ Stem extracts } & A-type trimer (45) & 16.9 & 18.2 & 15.4 & 14.6 & 14.1 & 19.5 \\
\hline & B-type trimer (46) & 14.3 & 13.3 & 13.9 & 15.8 & 18.1 & 11.3 \\
\hline & Procyanidin B2 (32) & 7.8 & 9.2 & 9.4 & 9.7 & 10.4 & 7.7 \\
\hline & 5-O-Caffeoylquinic-acid (18) & 6.9 & 7.5 & 6.4 & 8.0 & 7.0 & 4.6 \\
\hline & (-)-Epigallocatechin (15) & 2.9 & 2.7 & 3.3 & 2.9 & 2.6 & 5.0 \\
\hline & (-)-Epicatechin (34) & 3.1 & 4.7 & 7.3 & 4.9 & 6.1 & 7.4 \\
\hline & Quercetin-3-O-galactoside (62) & 4.8 & 2.8 & 3.6 & 2.4 & 1.8 & 2.2 \\
\hline & Quercetin hexuronide (64) & 4.4 & 3.2 & 2.4 & 7.1 & 6.2 & 2.2 \\
\hline & p-Coumaroyl malonylhexoside (96) & 6.4 & 5.9 & 6.2 & 6.4 & 4.8 & 7.0 \\
\hline & p-Coumaroyl monotropein (70) & 2.5 & 2.9 & 2.0 & 2.0 & 2.2 & 1.5 \\
\hline
\end{tabular}

a Values in parentheses correspond to compound number in Table 1.

b Mean for $n=3$.

from July and September (59 and $\mathbf{6 0}$ ) and fruits (99), as found for (-)-epicatechin. They thus appear to be specifically biosynthesised from spring to summer.

\subsubsection{Anthocyanins}

Both aqueous and ethanolic fruit extracts contained high levels of anthocyanins (Table 2). Fifteen anthocyanins were assessed through their major fragment ions at $m / z$ 303, 287, 317, 301, and 331, which are characteristic of the aglycones delphinidin, cyanidin, petunidin, peonidin, and malvidin, respectively (Table 5, Supplementary material). Delphinidin-3-O-galactoside and delphinidin-3-O-glucoside are predominant anthocyanins in fruit extracts (Može et al., 2011; Prencipe, Bruni, Guerrini, Benvenuti, \& Pellati, 2014).

\subsection{Influence of the harvest period on the phenolic composition in bilberry leaves, stems and fruits}

Relatively similar phenolic profiles were found in bilberry leaves and stems, harvested at the three different periods during the two years. Qualitative analysis showed the presence of caffeic acid and coumaric acid derivatives, quercetin glycosides, and (-)epicatechin in leaves, whereas in stems (-)-epigallocatechin and epicatechin-derived oligomers were additionally identified (Table 1).

In leaves, caffeic acid derivatives were the most representative group of phenolic compounds, as found earlier by Martz et al., 2010. Whatever the period and the year of harvest, their level ranged between 67 and $79 \%$ of the dry extract weight (Table 2). Their seasonal evolution differed between years 2013 and 2014. Chlorogenic acid (18) contributed more than half and its relative content varied between 55\% (May 2013) and 75\% (July 2013) (Table 3).

Flavonol glycosides were present in lesser contents in May leaves compared to $p$-coumaric acid derivatives although the opposite was observed in July and September. As a matter of fact, flavonol glycosides markedly increased in July and this high level remained steady (2013) or decreased (2014) in September. Liu et al. (2014) also observed no flavonol variation between July and September 2013 for leaves collected in Finland, while Martz et al. (2010) observed a slight increase for leaves exposed to light also in Finland (2006). The second most important contributor to the leaf extract was a quercetin hexuronide (64) except for May 2014, with relative levels ranging between 5\% (May 2014) and $12 \%$ (September 2014). Ranking third was quercetin-3-Ogalactoside (62) from July to September in year 2013 and July 2014 (3-8\%). The second and third leaf contributors are thus variable: ranking second in May 2014 and third in May 2013 is p-coumaroyl diacetylhexoside (78) (5\%) while p-coumaroyl malonylhexosides (83 and $\mathbf{9 6}$ ) are equally placed third in September 2014 (2.4\%).

With $p$-coumaric acid derivatives at their highest levels in May, another important contributor is p-coumaroyl monotropein (70) ranking 4th in May 2013 and May 2014, although this ranking largely decreased in July and September in both 2013 and 2014.

Finally, flavanol monomers and oligomers, which were mainly composed of (-)-epicatechin, cinchonain I (60) and cinchonain II (59), became quantifiable in July and September although in trace amounts.

In stems, flavanol oligomers were the major group, representing between 54 to $62 \%$ of the Sum of phenolic compounds (w/w of DE). The major contributor to the stem extract was an A-type trimer (45) in May, July, and September 2013 as well as in September $2014(15-20 \%)$ or a B-type trimer (46) in May and July 2014 (16-18\%). Ranking second was the same B-type trimer (46) for May, July, and September 2013 as well as for September 2014 (11-14\%) while the A-type trimer (45) was favored for May and July 2014 (14-15\%). Ranking third was dimer B2 (32) for all seasons over the two years of study with contents varying between 8 and 10\%. Furthermore, 5-caffeoylquinic acid (18) was the fourth more abundant compound from May to July whatever the year with levels between 7 and $8 \%$ when (-)-epicatechin (34) dominated in September (7\%). Finally, the next compounds highly present were: p-coumaroyl malonylhexoside (96), quercetin hexuronide (64), quercetin-3-O-galactoside (62), p-coumaroyl 
monotropein (70), and (-)-epigallocatechin (15). The recurrence for the three most abundant contributors over the seasons and years points to a flavanol oligomer biosynthesis under genetic control.

By contrast, seasonal and inter-annual variations were observed for contents in most phenolic groups in stem as in leaf (Table 2). The year effect was even higher for stems than for leaves, suggesting that abiotic stress clearly influences polyphenol biosynthesis. This can be attributed to contrasted weather conditions like air and soil temperature, hours of sunshine, and level of precipitations (Table 6, Supplementary material) as observed earlier by Martz et al. (2010) and Uleberg et al. (2012). Biotic stress was recently shown to elicit the biosynthesis of leaf phenolic compounds whose structures differed after attack by a fungal endophyte or $B$. cinerea (Koskimäki et al., 2009).

In fruits, the sum of phenolic compounds was lower than in leaves and stems and this could be attributed to the high sugar concentration in fruits. In both fruit extracts, anthocyanins clearly dominated with levels ranging from 22 to $35 \mathrm{mg} / \mathrm{g}$ DE and representing $83-85 \%$ of the extract weight (Table 2). The other classes ranked as follows in a decreasing order: caffeic acid derivatives (2.4-3.5 mg/g) > coumaric acid derivatives $(1.1-1.5 \mathrm{mg} / \mathrm{g})>$ flavonol glycosides $(0.9-1.4 \mathrm{mg} / \mathrm{g})>$ flavanol monomers $(0-0.1 \mathrm{mg} / \mathrm{g})$. The contents of these various phenolic classes remained relatively unaffected between 2013 and 2014.

\subsection{Characterization of flavan-3-ol oligomers}

In leaves, flavanol oligomers could appear as the second most abundant class of phenolic compounds when considering data from thioacidolysis (Table 4), ranging between 13 and $32 \mathrm{mg} / \mathrm{g}$ DE in July and September 2013 and 2014. This is rather contradictory with data from UPLC (Table 2) where no A-type or B-type oligomers, but only cinchonain II (59) were quantified in leaves (1.1$1.9 \mathrm{mg} / \mathrm{g} \mathrm{DE}$ ) for the same periods. mDP ranging from 2.9 to 4.5 is however in agreement with the presence of the eight B-type dimers and trimers identified by MS. Cinchonains II are expected to react in thioacidolysis partly releasing quantifiable (epi)catechin terminal units along with new benzylthioether adducts escaping quantitation. Of note, (-)-epicatechin was the only constituting unit of flavanol oligomers in leaves.

In stems, the contents in flavanol oligomers were similar whatever the method used, ranging between 48 and $70 \mathrm{mg} / \mathrm{g}$ DE after thioacidolysis and between 40 and $71 \mathrm{mg} / \mathrm{g}$ DE by UPLC (Tables 2 and 4). Moreover, seasonal variations for flavanol oligomers were close in 2013 and 2014, independently of the methods. However, the oligomer contents appear to be underestimated after thioacidolysis in accordance with A-type oligomers being incompletely degraded as well as (epi)gallocatechin units escaping quantification. Indeed, the major contributor to stems is an A-type trimer (45). $\mathrm{mDP}$ ranging between 2 and 3 are consistent with the predominance of two A-type and B-type trimers ( 45 and 46 ) by UPLC. Lastly, catechin appeared as both terminal units (2-8\%) and extension units $(1-3 \%)$.

In fruits, low amounts of flavanol oligomers (2-5 mg/g DE) were determined by the thioacidolysis method when no oligomers were quantified by UPLC, mostly because of co-elution with anthocyanins. (+)-Catechin appeared only as a terminal unit when (-)epicatechin was present both as terminal and extension units, accounting for more than $90 \%$ of the total units. mDP remained low (2-3). As expected, 55\% aqueous ethanol was twice as efficient at extracting oligomeric flavanols.

\subsection{Antioxidant activity of bilberry extracts}

The antioxidant activity of the bilberry extracts was determined by two complementary methods. The DPPH test relies on the ability of reducing molecules to transfer an electron or a hydrogen atom to the nitrogen-centred DPPH radical. As for the FolinCiocalteu method, it measures the ability of a sample to reduce transition metal ions as in the complex between sodium phosphomolybdate and phosphotungstate, giving access to the total phenolic content (TPC).

The TPC was reported in weight per dry matter for years 2013 and 2014 and in weight per dry extract in 2014 (Table 2). The difference is due to the extraction yield of the DM (ca. 58, 52, and 85\% $w / w$ for leaves, stems and fruits, respectively). When expressed in

Table 4

Flavan-3-ol composition and mDP in bilberry leaves, stems and fruits using thioacidolysis.

\begin{tabular}{|c|c|c|c|c|c|c|c|}
\hline \multirow{3}{*}{$\begin{array}{l}\text { Morphological part } \\
\text { extracts }\end{array}$} & \multirow[t]{3}{*}{ Period of vegetation } & \multicolumn{4}{|c|}{ Oligomeric flavanol characterization } & \multirow{3}{*}{$\begin{array}{l}\text { Flavanol } \\
\text { oligomers } \\
\text { (mg/g DE) }\end{array}$} & \multirow[t]{3}{*}{$\mathrm{mDP}$} \\
\hline & & \multicolumn{2}{|c|}{ Terminal units (\%) } & \multicolumn{2}{|c|}{ Extension units (\%) } & & \\
\hline & & $C A T$ & $E C$ & $C A T$ & $E C$ & & \\
\hline \multicolumn{8}{|l|}{ Leaf extracts } \\
\hline & May 2013 & - & $34.7 \pm 4.5$ & - & $65.3 \pm 16.6$ & $4.25 \pm 0.99(\mathrm{~A})$ & $2.9 \pm 0.5(\mathrm{~A})$ \\
\hline & May 2014 & - & $34.5 \pm 5.5$ & - & $65.5 \pm 2.2$ & $2.11 \pm 0.56(a)^{*}$ & $3.0 \pm 0.4(a)$ \\
\hline & July 2013 & $1.98 \pm 0.03$ & $27.9 \pm 5.8$ & - & $70.1 \pm 2.3$ & $32.4 \pm 2.2(\mathrm{~B})$ & $3.4 \pm 0.4(\mathrm{~A})$ \\
\hline & July 2014 & - & $22.1 \pm 0.3$ & - & $77.9 \pm 1.2$ & $25.5 \pm 1.4(b)^{*}$ & $4.5 \pm 0.1(b)^{*}$ \\
\hline & September 2013 & - & $26.3 \pm 2.3$ & - & $73.7 \pm 0.8$ & $23.8 \pm 0.6(C)$ & $3.8 \pm 0.2(\mathrm{~A})$ \\
\hline & September 2014 & - & $28.0 \pm 3.7^{*}$ & - & $72.0 \pm 1.7^{*}$ & $12.7 \pm 0.6(c)^{*}$ & $3.6 \pm 0.3(a)$ \\
\hline \multicolumn{8}{|l|}{ Stem extracts } \\
\hline & May 2013 & $2.17 \pm 0.11$ & $36.1 \pm 1.8$ & - & $61.7 \pm 3.0$ & $51.7 \pm 2.1(\mathrm{~A})$ & $2.6 \pm 0.0(\mathrm{~A})$ \\
\hline & May 2014 & - & $32.6 \pm 0.1^{*}$ & $1.61 \pm 0.37$ & $65.8 \pm 0.4^{*}$ & $60.0 \pm 4.3(a)^{*}$ & $3.1 \pm 0.0(\mathbf{a})^{*}$ \\
\hline & July 2013 & $3.10 \pm 0.87$ & $37.5 \pm 1.1$ & - & $59.4 \pm 0.4$ & $63.6 \pm 0.3(\mathrm{~B})$ & $2.5 \pm 0.1(\mathrm{~B})$ \\
\hline & July 2014 & - & $33.7 \pm 0.6^{*}$ & $1.20 \pm 0.17$ & $65.1 \pm 0.9^{*}$ & $69.6 \pm 2.3(b)^{*}$ & $3.0 \pm \mathbf{0 . 0}(\mathbf{b})^{*}$ \\
\hline & September 2013 & $5.86 \pm 1.06$ & $36.5 \pm 1.0$ & - & $57.6 \pm 1.9$ & $60.3 \pm 0.3(C)$ & $2.4 \pm 0.0(\mathrm{C})$ \\
\hline & September 2014 & $7.90 \pm 0.28$ & $38.1 \pm 0.9$ & $2.66 \pm 2.60$ & $51.4 \pm 2.0^{*}$ & $47.6 \pm 1.0(c)^{*}$ & $2.2 \pm 0.1(\mathbf{c})^{*}$ \\
\hline \multicolumn{8}{|l|}{ Fruit extracts } \\
\hline \multirow[t]{2}{*}{$\mathrm{H}_{2} \mathrm{O}$} & July 2013 & $9.48 \pm 2.61$ & $34.6 \pm 2.0$ & - & $55.9 \pm 23.5$ & $2.21 \pm 0.68$ & $2.3 \pm 0.6$ \\
\hline & July 2014 & $9.63 \pm 0.62$ & $37.6 \pm 0.3^{*}$ & - & $52.8 \pm 0.9$ & $2.45 \pm 0.06$ & $2.1 \pm 0.0$ \\
\hline \multirow[t]{2}{*}{ EtOH 55\% } & July 2013 & $8.99 \pm 2.50$ & $33.6 \pm 4.5$ & - & $57.4 \pm 28.0$ & $4.18 \pm 1.50$ & $2.3 \pm 0.5$ \\
\hline & July 2014 & $5.75 \pm 0.33$ & $27.1 \pm 1.0$ & - & $67.2 \pm 2.1$ & $5.42 \pm 0.25$ & $3.0 \pm 0.0^{*}$ \\
\hline
\end{tabular}

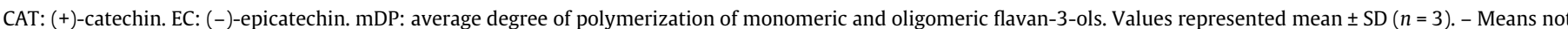

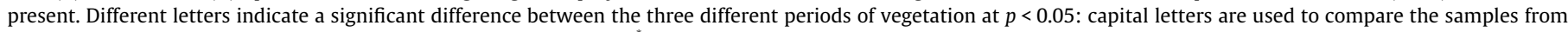
2013 and small letters are used to compare the samples from 2014. Means a significant difference between the two years $(p<0.05)$. 
mg GAE/g DE (year 2014), the TPC values were in the same range or higher than those found by summing all the phenolic compounds quantified by UPLC. As a matter of fact, correlation plots with stem, leaf and fruit data showed that TPC $(w / w$ of DM) were well correlated to the sum of phenolic compounds ( $w / w$ of DE) with $R^{2}$ of 0.73 and 0.62 except for leaves from May 2013 and 2014 (Fig. 2, Suppl. material). The removal of the May data markedly increased the correlation $\left(R^{2} 0.96\right.$ and 0.98$)$. This suggests a high correlation between these two methods when assaying bilberry phenolic compounds in all the morphological parts. May leaves presented unexpectedly low TPC when compared to TPC of the samples from July and September. It is worth noting that $p$-coumaric acid derivatives contribute to $20 \%$ of the phenolic pool in May and only $7 \%$ in July and September. Besides, $p$-coumaric acid derivatives remained at low levels in stems (11-14\%) and fruits (4-5\%) from May to September. As a matter of fact, $p$-coumaric acid was 2.5 -fold less reactive than caffeic acid with the Folin Ciocalteu reagent (Ma \& Cheung, 2007). Moreover, the relative reactivity of gallic acid (1.0), caffeic acid (0.96), chlorogenic acid (1.36), and rutin (1.53) was higher than that of salicylic acid (0.26) and tyrosine (0.38), which are structurally related to $p$-coumaric acid (Everette et al., 2010).

The DPPH-scavenging activities of bilberry extracts showed contrasting seasonal variations between 2013 and 2014 (Fig. 1). A significant increase in leaf antioxidant activity is exhibited in July and September 2013 when this increase was only observed in July 2014. The antioxidant activity of the stem extracts was less affected by the season. As to fruits, the use of ethanol-containing solvents significantly improved the recovery in phenolic compounds and the antioxidant activity by both TPC and DPPH tests in 2013 while this effect was modest in 2014. Finally, the annual effect on the DPPH antioxidant activities is similar to that exhibited for the TPC values. Additionally, the activity in the DPPH test and the sum of phenolic compounds $(w / w$ of $\mathrm{DE})$ were highly correlated, with $R^{2}$ of 0.70 and 0.77 in 2013 and 2014 for all eight samples (Fig. 2, Suppl. material). Moreover, the correlation plots are similar to the ones observed for sum of phenolic compounds vs TPC. The lower reactivity of May leaves is likely linked to its large content in $p$-coumaric acid derivatives. Phenolic compounds displaying a dihydroxyphenyl moiety are generally more antioxidant than those containing a monohydroxyphenyl moiety.

Finally, TPC strongly correlated with the DPPH radical scavenging activity with $R^{2}$ of 0.91 and 0.94 for samples from 2013 and 2014, respectively (Fig. 2, Suppl. material). This suggests that phenolic compounds with mono- and dihydroxyphenyl moieties as well as other reducing substances present in the extract display the same reducing ability towards transition metal ions as in the Folin Ciocalteu method and N-centred radicals as in the DPPH test.

\section{Conclusions}

This study reports the most comprehensive qualitative analysis ever conducted on bilberry leaves, stems, and fruits, leading to the identification of 106 phenolic compounds. In particular, structures were proposed for 46 new compounds.

Quantitative analysis allowed the accurate determination of the ten to twelve most important contributors belonging to the groups caffeoyl derivatives, $p$-coumaroyl derivatives, flavonol glycosides, anthocyanins, and flavanol monomers and oligomers. In general, the ranking of the major contributors in leaf and stem showed low or no seasonal variations, respectively. Some variations were however outlined between May and July suggesting the appearance (flavanol oligomers in leaves, cinchonains I and II in stems from July and September) or disappearance of minor compounds ( $p$-coumaroyl derivatives in leaves from May). The intra-annual variations for the content in the various phenolic groups generally differed between 2013 and 2014, in agreement with biotic and abiotic stresses. Finally, the phenolic content was highly correlated to the antioxidant activity in leaf, stem and fruit extracts of bilberry.

All the morphological parts of bilberry proved to be suitable for valorisation as sources of natural phenolic compounds. Regarding the period of harvest, leaves and stems should be better collected in July or September to be valuable feedstocks for the production of food supplements. May leaves are of interest if the health benefit of $p$-coumaroyl derivatives is sought. The stability of the collected dry material remains however to be assessed, to determine its optimal shelf life.

\section{Conflict of interest statement}

The authors declare no conflict of interest.

\section{Acknowledgements}

O. C. Bujor thanks the Erasmus+ Programme for student mobility grants. We gratefully thank Mrs Camelia Mihăilescu (Faculty of Chemical Engineering and Environmental Protection, Iasi, Romania) and Mrs Michèle Loonis (INRA-SQPOV Unit, Avignon, France) for their respective guidance in plant extraction and UPLC/MS analyses, respectively. We also thank Dr. Gina Tiron from National Meteorological Administration-Moldova Meteorological Center of Iasi, for providing the meteorological data.

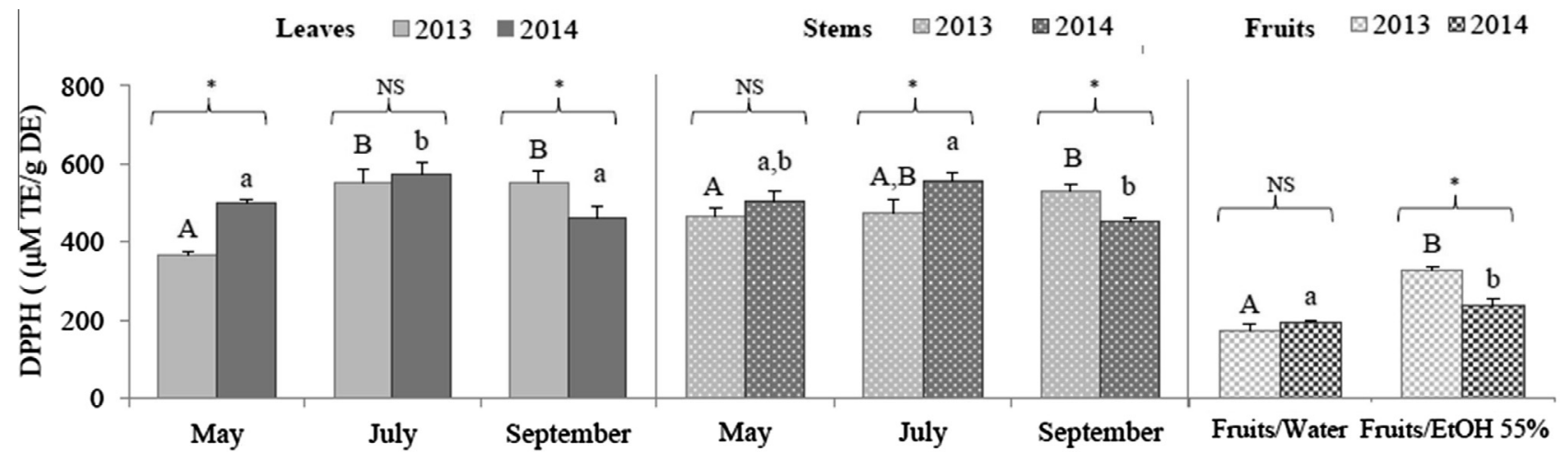

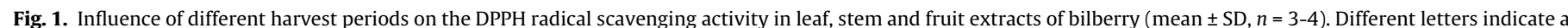

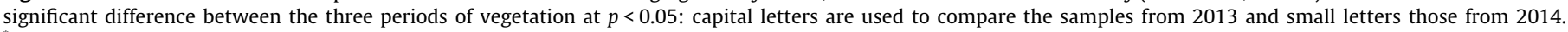
* Means a significant difference between the two years with $p<0.05$. NS; not significant. 


\section{Appendix A. Supplementary data}

Supplementary data associated with this article can be found, in the online version, at http://dx.doi.org/10.1016/j.foodchem.2016. 06.042 .

\section{References}

Akerström, A., Jaakola, L., Bång, U., \& Jäderlund, A. (2010). Effects of latitude-related factors and geographical origin on anthocyanidin concentrations in fruits of Vaccinium myrtillus L. (bilberries). Journal of Agricultural and Food Chemistry, 58, $11939-11945$.

Bujor, O.-B., Talmaciu, I. A., Volf, I., \& Popa, I. V. (2015). Biorefining to recover aromatic compounds with biological properties. Tappi Journal, 14(3), 187-193.

Chanforan, C., Loonis, M., Mora, N., Caris-Veyrat, C., \& Dufour, C. (2012). The impact of industrial processing on health-beneficial tomato microconstituents. Food Chemistry, 134, 1786-1795.

Clifford, M. N., Johnston, K. L., Knight, S., \& Kuhnert, N. (2003). Hierarchical scheme for LC-MSn identification of chlorogenic acids. Journal of Agricultural and Food Chemistry, 51, 2900-2911.

Erlund, I., Koli, R., Alfthan, G., Marniemi, J., Puukka, P., Mustonen, P., Mattila, P., et al. (2008). Favorable effects of berry consumption on platelet function, blood pressure, and HDL cholesterol. The American Journal of Clinical Nutrition, 87, 323-331.

Everette, J. D., Bryant, Q. M., Green, A. M., Abbey, Y. A., Wangila, G. W., \& Walker, R. B. (2010). A thorough study of reactivity of various compounds classes towards the Folin-Ciocalteu reagent. Journal of Agricultural and Food Chemistry, 58(14), 8139-8144.

Goupy, P., Dufour, C., Loonis, M., \& Dangles, O. (2003). Quantitative kinetic analysis of hydrogen transfer reactions from dietary polyphenols to the DPPH radical. Journal of Agricultural and Food Chemistry, 51(3), 615-622.

Hainal, A. C., Ignat, I., Volf, I., \& Popa, I. V. (2011). Transformation of polyphenols from biomass by some yeast species. Cellulose Chemistry and Technology, 45(34), 211-219.

Hokkanen, J., Mattila, S., Jaakola, L., Pirttilä, A. M., \& Tolonen, A. (2009). Identification of polyphenols from lingonberry (Vaccinium vitis-idaea L.), bilberry (Vaccinium myrtillus L.) and hybrid bilberry (Vaccinium $x$ intermedium Ruthe L.) leaves. Journal of Agricultural and Food Chemistry, 57, 9437-9447.

Ieri, F., Martini, S., Innocenti, M., \& Mulinacci, N. (2013). Phenolic distribution in liquid preparations of Vaccinium myrtillus L. and Vaccinium vitis idaea L. Phytochemical Analysis, 24, 467-475.

Jovančević, M., Balijagić, J., Menković, N., Šavikin, K., Zdunić, G., Janković, T., \& DekićIvanković, M. (2011). Analysis of polyphenols in wild populations of bilberry (Vaccinium myrtillus L.) from Montenegro. Journal of Medicinal Plants Research, 5 (6), 910-914.

Koskimäki, J. J., Hokkanen, J., Jaakola, L., Suorsa, M., Tolonen, A., Mattila, S., Pirttila, A. M., et al. (2009). Flavonoid biosynthesis and degradation play a role in early defence responses of bilberry (Vaccinium myrtillus) against biotic stress. European Journal of Plant Pathology, 125, 629-640.

Lätti, A. K. Jaakola, L., Riihinen, K. R. \& Kainulainen, P. S. (2010). Anthocyanin and flavonol variation in bog bilberries (Vaccinium uliginosum L.) in Finland. Journal of Agricultural and Food Chemistry, 58, 427-433.

Le Bourvellec, C., Bouzerzour, K., Ginies, C., Regis, S., Plé, Y., \& Renard, M. G. C. C. (2011). Phenolic and polysaccharidic composition of applesauce is close to that of apple flesh. Journal of Food Composition and Analysis, 24, 537-547.
Liu, P., Lindstedt, A., Markkinen, N., Sinkkonen, J., Suomela, J.-P., \& Yang, B. (2014). Characterization of metabolite profiles of leaves of bilberry (Vaccinium myrtillus L.) and lingonberry (Vaccinium vitis-idaea L.). Journal of Agricultural and Food Chemistry, 62, 12015-12026.

Ma, Y.-T., \& Cheung, P. C. K. (2007). Spectrophotometric determination of polyphenols by enzymatic and chemical methods - a comparison of structure-activity relationship. Journal of Agricultural and Food Chemistry, 55, $4222-4228$

Mane, C., Loonis, M., Juhel, C., Dufour, C., \& Malien-Aubert, C. (2011). Food grade lingonberry extract: polyphenolic composition and in vivo protective effect against oxidative stress. Journal of Agricultural and Food Chemistry, 59, 3330-3339.

Martz, F., Jaakola, L., Julkunen-Tiitto, R., \& Stark, S. (2010). Phenolic composition and antioxidant capacity of bilberry (Vaccinium myrtillus) leaves in Northern Europe following foliar development and along environmental gradients. Journal of Chemical Ecology, 36, 1017-1028.

Mauray, A., Felgines, C., Morand, C., Mazur, A., Scalbert, A., \& Milenkovic, D. (2010). Nutrigenomic analysis of the protective effects of bilberry anthocyanin-rich extract in apo E-deficient mice. Genes Nutrition, 5, 343-353.

Mikulic-Petkovsek, M., Schmitzer, V., Slatnar, A., Stampar, F., \& Veberic, R. (2015). A comparison of fruit quality parameters of wild bilberry (Vaccinium myrtillus L.) growing at different locations. Journal of the Science of Food and Agriculture, 95, 776-785.

Može, Š., Polak, T., Gašperlin, L., Koron, D., Vanzo, A., Poklar Ulrih, N., \& Abram, V. (2011). Phenolics in slovenian bilberries (Vaccinium myrtillus L.) and blueberries (Vaccinium corymbosum L.). Journal of Agricultural and Food Chemistry, 59(13), 6998-7004.

Mykkänen, O. T., Huotari, A., Herzig, K.-H., Dunlop, T. W., Mykkänen, H., \& Kirjavainen, P. V. (2014). Wild blueberries (Vaccinium myrtillus) alleviate inflammation and hypertension associated with developing obesity in mice fed with a high-fat diet. PLOS ONE, 9(12), 1-21.

Prencipe, F. P., Bruni, R., Guerrini, A., Rossi, D., Benvenuti, S., \& Pellati, F. (2014). Metabolite profiling of polyphenols in Vaccinium berries and determination of their chemopreventive properties. Journal of Pharmaceutical and Biomedical Analysis, 89, 257-267.

Rouanet, J. M., Décordé, K., Del Rio, D., Auger, C., Borges, G., Cristol, J.-P., Lean, M. E. J., et al. (2010). Berry juices, teas, antioxidants and the prevention of atherosclerosis in hamsters. Food Chemistry, 118(2), 266-271.

Teleszko, M., \& Wojdyło, A. (2015). Comparison of phenolic compounds and antioxidant potential between selected edible fruits and their leaves. Journal of Functional Foods, 14, 736-746.

Uleberg, E., Rohloff, J., Jaakola, L., Trôst, K., Junttila, O., Häggman, H., \& Martinussen, I. (2012). Effects of temperature and photoperiod on yield and chemical composition of northern and southern clones of bilberry (Vaccinium myrtillus L.). Journal of Agricultural and Food Chemistry, 60, 10406-10414.

Vučić, D. M., Petković, M. R., Rodić-Grabovac, B. B., Stefanović, O. D., Vasić, S. M., \& Čomić, L. R. (2013). Antibacterial and antioxidant activities of bilberry (Vaccinium myrtillus L.) in vitro. African Journal of Microbiology Research, 7(45), 5130-5136.

Watrelot, A. A., Renard, C. M. G. C., \& Le Bourvellec, C. (2015). Comparison of microcalorimetry and haze formation to quantify the association of B-type procyanidins to poly-L-proline and bovine serum albumin. LWT-Food Science and Technology, 63, 376-382.

Zheng, X., Xu, X., Liu, C., Sun, Y., Lin, Z., \& Liu, H. (2013). Extraction characteristics and optimal parameters of anthocyanin from blueberry powder under microwave-assisted extraction conditions. Separation and Purification Technology, 104, 17-25. 